\title{
On the concept and theory of induced drag for viscous and incompressible steady flow
}

EP

Cite as: Phys. Fluids 31, 065106 (2019); https://doi.org/10.1063/1.5090165

Submitted: 25 January 2019 . Accepted: 09 May 2019. Published Online: 10 June 2019

Shu-Fan Zou (邹舒帆) (D, J. Z. Wu (吴介之), An-Kang Gao (高安康) (D, Luoqin Liu (刘罗勤) (D, Linlin Kang (康林林), and Yipeng Shi (史一蓬)

\section{COLLECTIONS}

EP This paper was selected as an Editor's Pick
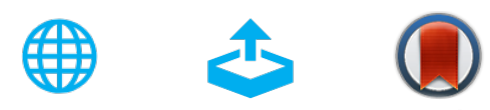

\section{ARTICLES YOU MAY BE INTERESTED IN}

Control effect of micro vortex generators on attached cavitation instability

Physics of Fluids 31, 064102 (2019); https://doi.org/10.1063/1.5099089

An experimental study of the dynamic aerodynamic characteristics of a yaw-oscillating wind turbine airfoil

Physics of Fluids 31, 067102 (2019); https://doi.org/10.1063/1.5088854

Modeling of mass transfer enhancement in a magnetofluidic micromixer

Physics of Fluids 31, 063603 (2019); https://doi.org/10.1063/1.5093498

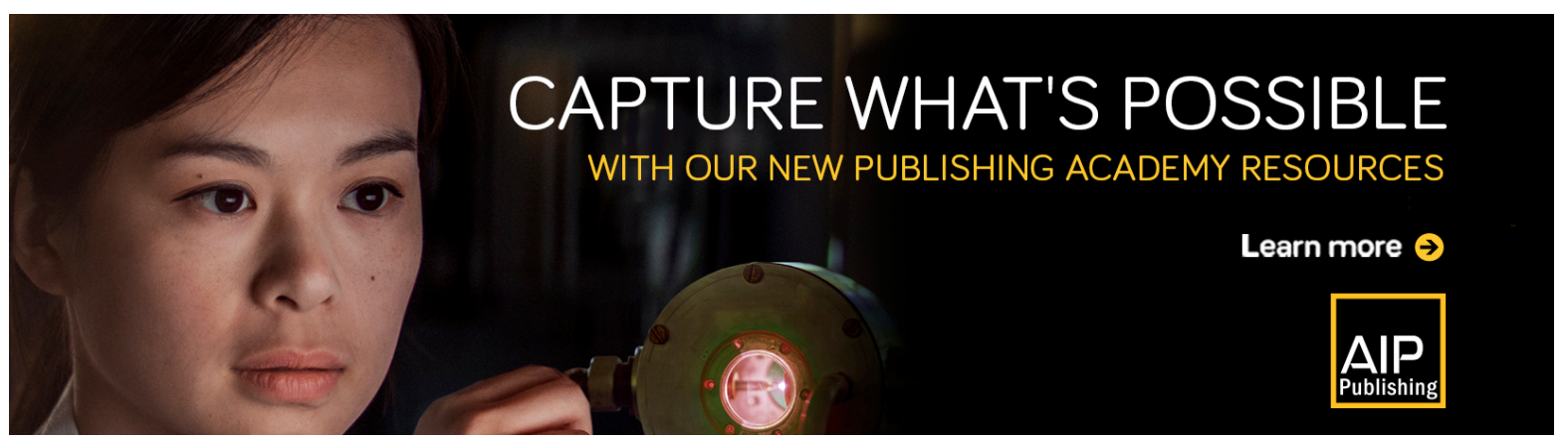




\title{
On the concept and theory of induced drag for viscous and incompressible steady flow
}

Cite as: Phys. Fluids 31, 065106 (2019); doi: 10.1063/1.5090165

Submitted: 25 January 2019 - Accepted: 9 May 2019 •

Published Online: 10 June 2019

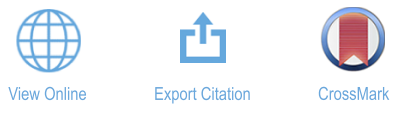

\author{
Shu-Fan Zou (邹舒帆), ' (D) J. Z. Wu (吴介之), ${ }^{1, a)}$ An-Kang Gao (高安康), ' (D) Luoqin Liu (刘罗勤), \\ Linlin Kang (康林林), ${ }^{7}$ and Yipeng Shi (史一蓬)
}

\begin{abstract}
AFFILIATIONS
${ }^{1}$ State Key Laboratory for Turbulence and Complex Systems, College of Engineering, Peking University, Beijing 100871, China

${ }^{2}$ Physics of Fluids Group, Faculty of Science and Technology, University of Twente, 7500AE Enschede, The Netherlands
\end{abstract}

a) Electronic mail: jzwu@coe.pku.edu.cn

\begin{abstract}
For steady flow, one usually decomposes the total drag into different components by wake-plane integrals and seeks their reduction strategies separately. Unlike the body-surface stress integral, the induced drag as well as the profile drag has been found to depend on the streamwise location of the wake plane used for drag estimate. It gradually diminishes as the wake plane moves downstream, which was often attributed to numerical dissipation. In this paper, we present an exact general force-breakdown theory and its numerical demonstrations for viscous incompressible flow over an arbitrary aircraft to address this puzzling issue. Based on the theory, the induced and profile drags do depend inherently on the wake-plane location rather than being merely caused by numerical dissipation. The underlying mechanisms are identified in terms of the components, moments, and physical dissipation of the Lamb-vector field produced by the aircraft motion. This theoretical prediction is fully consistent with the linear far-field force theory that the induced drag finally vanishes and the profile drag increases to the total drag at an infinitely far field for viscous flow. Moreover, as a product of this exact theory, a new compact midwake approximation for the induced drag is proposed for the convenience of routine wake survey in industry. Its prediction is similar to conventional formulas for attached flow but behaves much better for separated flow.
\end{abstract}

Published under license by AIP Publishing. https://doi.org/10.1063/1.5090165

\section{INTRODUCTION}

Drag reduction is one of the crucial fields of external flow aerodynamics. This subject has been an important target in aircraft design, of which a necessary premise is the rational decomposition of drag and its reliable prediction for a generic body configuration in a three-dimensional (3D) viscous flow. However, so far a full consensus of this issue has not been reached in literature and engineering community.

The drag decomposition can be rationally made in two alternative categories based on the body-surface stress integrals and domain integrals, respectively. In category 1 , the drag $D$ is split to the pressure drag $D_{p}$ and friction drag $D_{f}$. This splitting is fully free from any ambiguity. In category 2 and for time-averaged steady flow, the drag is divided into induced drag $D_{i}{ }^{1}$ and profile drag $D_{P}$, ${ }^{2,3}$ for which the domain integrals can be reduced to wake-plane integrals at a chosen streamwise location, to be denoted by $x$ in the windaxis coordinates $(x, y, z)$ with the origin at the aircraft leading edge.
The profile drag $D_{P}$ reflects the kinetic energy loss caused by viscous dissipation, which is an irreversible process and dominated by the streamwise evolution of the flow field along the wing. The induced drag $D_{i}$ reflects the kinetic energy carried away by the lifting vortices as they form a steady wake. It only involves the reversible process and is considered mainly determined by the spanwise variation of the circulation. In addition to these two pairs of drag components, $\left(D_{p}, D_{f}\right)$ and $\left(D_{P}, D_{i}\right)$, some more drag components have been introduced in engineering community (e.g., Refs. 4 and 5), but most of them remain at an empirical level with no rational theoretical definitions.

The relation between categories 1 and 2 is as follows: $D_{p}$ contains $D_{i}$ and the form drag, while $D_{f}$ is $D_{P}$ minus the form drag. ${ }^{6}$ The form drag is a "secondary component" depending strongly on the shape and attitude of the body (Ref. 7, p. 332). It equals pressure drag in two dimensions (2D). The pressure drag is difficult to be linked with local flow structures because the wall-pressure distribution links closely to the global flow field. In contrast, it is believed 
that the boundary-layer theory and trailing-vortex theory can be used to explain the sources of friction and induced drags, respectively, and these theories are necessary to optimize aerodynamic design and develop drag reduction strategies. Although $D_{f}$ and $D_{i}$ come from different categories via the body-surface stress integral and wake integral, respectively, they are treated as dominant sources of $\mathrm{drag}^{8}$ and main objectivities of engineering drag reduction.

As is well known, the total lift $L$ and drag $D$ are experimentally measurable fixed numbers solely depending on flow conditions and aircraft configurations. Within an acceptable error bound, the calculated or measured $L$ and $D$ are objective and reliable. The same objective nature exists for the decomposed $D_{p}$ and $D_{f}$ in category 1 . However, the situation for $D_{i}$ and $D_{P}$ in category 2 is different due to two congenital features of domain/wake integrals.

First, experiments cannot measure $D_{i}$ and $D_{P}$ separately; at least one of them has to be theoretically defined in advance (another can be derived from the known $D$ ). Thus, the reliability of the predicted $D_{i}$ and/or $D_{P}$ depends solely on that of their theoretical definitions. For instance, in computational fluid dynamics (CFD), researchers determine total drag, $D_{f}$, and $D_{p}$ using a Navier-Stokes solver. The induced drag is determined by using a Trefftz plane analysis based on the spanwise lift distribution, and then, the form drag follows by subtracting the induced drag from the pressure drag. This is one way they can get unique numbers for various drag components. An inaccurate or oversimplified defining theory for $D_{i}$ would cause the drag-reduction technology to deviate from the target, while by that definition, one even could not tell where things are going wrong. In addition, the exact definition and even existence of the Trefftz plane in a general flow are still a controversial issue.

Second, any force component in terms of domain/wake integrals may depend on the selected domain, in principle, so that $D_{i}$ and $D_{P}$ may generically be domain/wake-location dependent, denoted by domain $/ x$-dependency. Indeed, it has been gradually realized that as the streamwise location $x$ of the selected wake plane increases, $D_{i}$ and $D_{P}$ slowly change to keep $D\left(=D_{i}+D_{P}\right)$ be domain $/ x$-independent. They are functions of $x$ rather than fixed numbers. ${ }^{10-13}$ Moreover, via linear far-field force theory, Liu et al. ${ }^{14}$ have strictly proved that as the location $x$ of a wake plane $W$ recedes to infinity (entering the linearized and viscous steady far field), $D_{i}$ approaches zero, while only $D_{P}$ remains and becomes the total drag. The far-field force theory provides a valuable check point of any viscous theory of drag breakdown. Actually, some researchers have also found that $D_{i}$ gradually diminishes as $x$ increases, ${ }^{15-19}$ which, however, was attributed to be probably associated with the interchange of the vorticity to entropy caused by numerical dissipations. Thus, a modification by adding an increased entropy drag into the initial induced drag was proposed to reduce its variation with $x$. Owing to these controversies and artificial modifications, consequently, some researchers simply use $D$ minus $D_{P}$ to define the induced drag, ${ }^{20}$ and some others just care about the total drag $D$ only.

It has to be remarked here that, in this paper, a flow region will be said in the near field, midfield, or far field if the flow therein is highly nonlinear, is weakly nonlinear, or can be linearized, respectively. This terminology differs from that used in common aerodynamics literature.

In our view, category 2 has its unique potential for understanding the physical origin and developing the control strategy of $D_{i}$ and $D_{P}$. The concept of these two drag components does reflect different mechanisms of interaction between the flow field and the configuration. Thus, at the fundamental level, one should make every effort to clarify the theoretical concepts, obtain exact general formulas, and understand the underlying physics of $D_{i}$ and $D_{P}$, along with the very root of their possible domain $/ x$-dependency. Then, at the applied level, it is also necessary to clarify in which situation the domain $/ x$ dependency can be ignored, in practice, especially for the conventional aircraft design community that continues taking $D_{i}$ and $D_{P}$ as fixed numbers.

This paper is devoted to these tasks. In Sec. II, we first present a general and exact theory on the profile and induced drags for 3D incompressible, viscous, and steady flow over a generic body, in terms of domain/wake integrals. The theory is demonstrated by numerical simulation of the flows over two typical steady flow types: attached flow over an elliptic wing of large aspect ratios at small angles of attack and separated flow over a slender delta wing at large

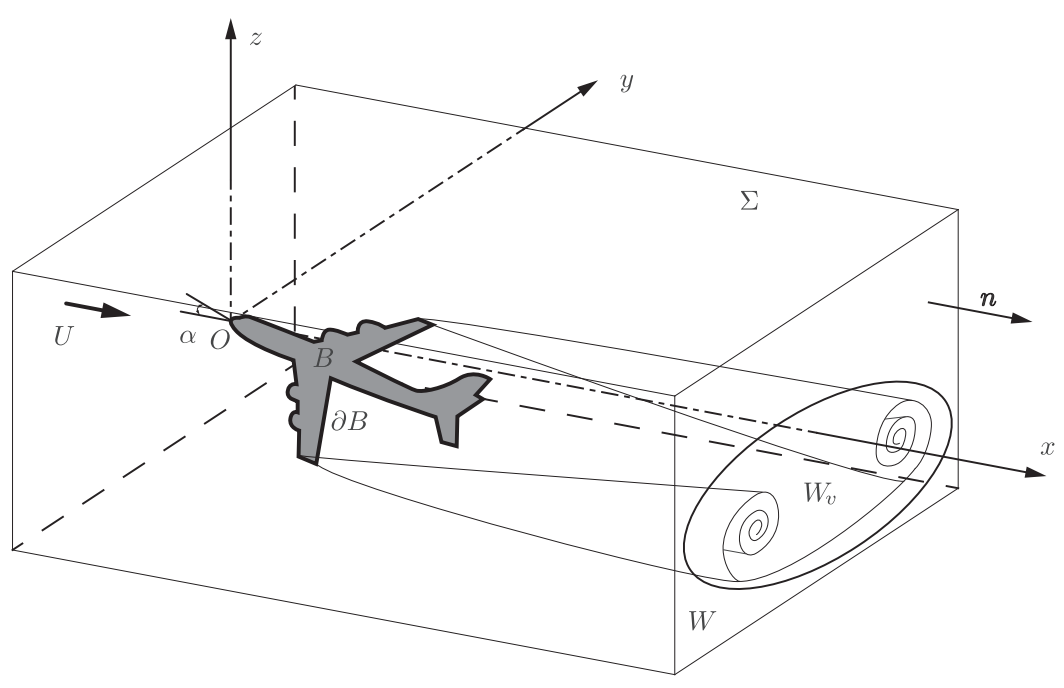

FIG. 1. Flow domain to be analyzed and notations. 
angles of attack. Although most of real flows are turbulent or transitional, for neatness the theory is formulated for laminar flow only-it can be easily modified to cover Reynolds-averaged turbulent flow by introducing turbulent viscosity. ${ }^{21-23}$ Unlike profile drag, the primary definition of induced drag is in terms of domain integral but will be cast to a maximally compact integral over a small vortical wake $W_{v}$ (Fig. 1). Interestingly, in the integral, some extra noncompact and explicitly $x$-dependent terms are inevitable.

In Sec. III, we focus on the precise dynamic root of the observed $x$-dependency of $D_{i}$ and $D_{P}$ by rigorous formulas of $\mathrm{d} D_{i} / \mathrm{d} x$ $=-\mathrm{d} D_{P} / \mathrm{d} x$. The underlying mechanisms of this $x$-dependency are identified in terms of the components, moments, and physical dissipation of the Lamb-vector field produced by the aircraft motion. This theoretical prediction is fully consistent with the linear far-field force theory that the induced drag finally vanishes and the profile drag increases to the total drag at an infinitely far field for viscous flow. Moreover, this study also reveals under what condition there is $\mathrm{d} D_{i} / \mathrm{d} x \simeq 0$, as demonstrated by numerical tests. This observation leads to a new compact midwake approximation of the exact $D_{i}$ formula, convenient for routine wake survey in industry, which is proposed in Sec. IV.

Concluding remarks and final discussion are made in Sec. V, and some technical details, both theoretical and numerical, are given in Appendixes A-C.

\section{GENERAL DRAG-BREAKDOWN THEORY BY DOMAIN/WAKE INTEGRALS}

We consider a 3D steady viscous and incompressible flow with uniform incoming velocity $\boldsymbol{U}=U \boldsymbol{e}_{x}$ over a generic stationary body configuration $B$ bounded by $\partial B$. Figure 1 defines the coordinate system $(x, y, z)$ fixed to the body and some notations used below. Here, $y$ and $z$ are along the spanwise and vertical-up directions, respectively. The control surface encloses a fluid volume $V_{f}$ that surrounds the solid body $B$ so that $V=V_{f} \cup B$ is the total volume bounded by $\Sigma$. The unit normal vector $\boldsymbol{n}$ of $\partial V_{f}=\Sigma \cup \partial B$ points out of the fluid. At large Reynolds numbers, viscous forces on $\Sigma$ can well be neglected, but we retain it in this section for completeness. The control surface $\Sigma$ cuts the vorticity field only at its downstream face; on and outside the front and side faces of $\Sigma$, the flow is inviscid and irrotational. The vortical wake with $\boldsymbol{\omega} \neq 0$ appears only in a small subset $W_{v}$ of a wake plane $W$.

We first make a critical revisit to the existing relevant theories as guidance to the follow-up discussions and then introduce an exact general theory. Numerical examples are displayed side by side with theoretical development.

\section{A. Available force theories based on domain/wake integrals}

The profile drag is defined by a wake-plane integral ${ }^{2,3}(W-$ integral for short)

$$
D_{P}=\int_{W}\left(P_{\infty}-P\right) \mathrm{d} S,
$$

where $P \equiv p+\rho|\boldsymbol{u}|^{2} / 2$ is the total pressure, and $p, \rho$, and $\boldsymbol{u}$ are the pressure, density, and velocity, respectively. In Sec. II B, we shall confirm that (1) is exact and applicable to generic steady flow over arbitrary configurations.
Although CFD/EFD can already well resolve many viscous complex flows and provide rich numerical/measured flow-field data, as described earlier they cannot tell what the induced-drag is. In current engineering applications, two simple formulas of $D_{i}$ are used as its definition. One is the classic formula of Prandtl ${ }^{1,24}$ for inviscid flow with $D_{P}=0$,

$$
C_{D i}=\frac{C_{L}^{2}}{\pi \Lambda}(1+\delta), \quad \delta=\sum_{n=2}^{\infty} n\left(\frac{A_{n}}{A_{1}}\right)^{2} \geq 0,
$$

where $\Lambda$ and $A_{n}$ 's are the wing aspect ratio and Fourier coefficients of the spanwise lift distribution, respectively. $\delta=0$ for the elliptical lift distribution yields the minimum induced drag for a planar wing system. The other is the formula of Maskell, ${ }^{25}$ supposed to be applicable to viscous flow,

$$
D_{i}=\frac{\rho}{2} \int_{T} \omega_{x} \psi_{x} \mathrm{~d} S=-\frac{\rho}{4 \pi} \int_{T} \int_{T} \omega_{x} \omega_{x}^{\prime} \log \left|\boldsymbol{r}-\boldsymbol{r}^{\prime}\right| \mathrm{d} S \mathrm{~d} S^{\prime},
$$

where $\omega_{x}$ is the streamwise vorticity, $\nabla^{2} \psi_{x}=-\omega_{x}$, and $\boldsymbol{r}=y \boldsymbol{e}_{y}+z \boldsymbol{e}_{z}$ is the position vector on a far-wake Trefftz plane $T$ characterized by assuming $\boldsymbol{\omega}=\left(\omega_{x}, 0,0\right)$. Both (2) and (3) have been found to work well for attached flow over a thin wing of large aspect ratio at small angles of attack, ${ }^{22}$ but there is still a gap between them and real complex flows.

How to define the induced drag for more general flows has been an active research area in the past three decades. $6,9,10,12,13,15,26,27$ In these discussions, another general formula of $D_{i}$ often appears as a starting point. By setting $\boldsymbol{u}=\boldsymbol{U}+\boldsymbol{v}=\left(U+u^{\prime}, v, w\right)$, there is $6,9,13,15,26,27$

$$
D_{i}=\rho \int_{W}\left(k-u^{\prime 2}\right) \mathrm{d} S=\frac{\rho}{2} \int_{W}\left(v^{2}+w^{2}-u^{\prime 2}\right) \mathrm{d} S,
$$

where $k=|\boldsymbol{v}|^{2} / 2$ is the disturbance kinetic energy. But, (4) provides no more than a link between the induced drag and the continuous downstream advection of disturbance kinetic energy subtracting the streamwise disturbance momentum flux. Since $k$ minus $u^{\prime 2}$ is widely spread in the wake and its physical meaning is vague, this formula is not recommended for practical wake measurements. Theoretical approaches (for compressible flow) were mostly of smallperturbation type, and (3) was proved to hold to the leading order. A comprehensive and comparative review of drag breakdown from both theoretical and experimental aspects is given by Méheut and Bailly. ${ }^{13}$ Despite of these efforts, however, no significant breakthrough has occurred for induced drag in fully viscous and nonlinear flow. Actually, it is here one encounters the key "long-standing dissatisfaction" of entire theoretical drag prediction. 'This situation explains specifically why $D_{i}$ is the very bottleneck problem.

\section{B. Primary definition of induced and profile drags in viscous flow}

For defining domain/ $W$-integral based drag components, one usually starts from the conventional momentum equation, of which (4) is one of the results. However, the most natural and simplest way to define $D_{P}$ and $D_{i}$ is to start from the general steady vortex-force theory, pioneered by Prandtl $^{1}$ one hundred years ago in the same seminal paper on the lifting-line theory and induced drag, the latter being the lowest-order simplification of the former (see Ref. 28). An easy extension of Prandtl's general theory to viscous flow has been given in Ref. 29 (Sec. 11.5), and we now tailor it to focus on 
defining the drag components. We start from the incompressible Crocco-Vazsonyi equation

$$
\rho \boldsymbol{\omega} \times \boldsymbol{u}=-\nabla\left(P-P_{\infty}\right)-\mu \nabla \times \boldsymbol{\omega},
$$

where $\omega$ and $\mu$ are vorticity and viscosity, respectively, and the constant $P_{\infty}$ is inserted for clarity. The gradient of total-pressure variation is the naturally obtainable maximum longitudinal (curlfree) term. For Reynolds-averaged turbulent flow, one can just introduce an eddy viscosity $\mu_{t}$ to modify the molecular viscosity $\mu$, and the governing equation is formally the same as (5). ${ }^{21,22}$ With this in mind and for neatness, in the following discussions, we shall focus on the general laminar $\mathrm{N}-\mathrm{S}$ equation.

The drag comes from the $x$-component of (5),

$$
\boldsymbol{e}_{x} \cdot \rho(\boldsymbol{v} \times \boldsymbol{\omega})=-\nabla \cdot\left[\left(P_{\infty}-P\right) \boldsymbol{e}_{x}+\mu \boldsymbol{\omega} \times \boldsymbol{e}_{x}\right],
$$

from which the primary definitions of $D_{i}$ and $D_{P}$ naturally follow from integrating its left-hand side over the fluid $V_{f}$ and casting its right-hand side to the boundary integral over $\partial V_{f}=\Sigma \cup \partial B$. Specifically, the boundary integral over $\Sigma$ yields exactly the same profile drag as given by (1), and the domain integral of the left-hand side of (6) yields the primary and exact definition of the induced drag

$$
D_{i}=\rho \int_{V} \boldsymbol{e}_{x} \cdot(\boldsymbol{v} \times \boldsymbol{\omega}) \mathrm{d} V,
$$

as first proposed by $\mathrm{Wu}, \mathrm{Ma}$, and $\mathrm{Zhou}^{29}$ and then numerically verified by Marongiu, Tognaccini, and Ueno. ${ }^{22}$ As usual, the front and side faces of $\Sigma$ can be chosen so that not only the flow thereon is irrotational with $\boldsymbol{\omega}=0$ and $P=P_{\infty}$ but also all disturbance quantities of $O\left(|\boldsymbol{v}|^{2}\right)$ are negligible. Then, by identity (A4), (7) is cast to a $W$-integral at once, which is nothing but exactly (4).

Meanwhile, recall that the standard force formula by the surface-stress integral reads

$$
\boldsymbol{F}=-\int_{\partial B}(-p \boldsymbol{n}+\mu \boldsymbol{\omega} \times \boldsymbol{n}) \mathrm{d} S .
$$

Its $x$-component now comes from integrating (6) over $\partial B$, which yields $D_{p}$ and $D_{f}$,

$$
D_{p}=\int_{\partial B} p \boldsymbol{n} \cdot \boldsymbol{e}_{x} \mathrm{~d} S, \quad D_{f}=-\int_{\partial B} \boldsymbol{e}_{x} \cdot(\mu \boldsymbol{\omega} \times \boldsymbol{n}) \mathrm{d} S .
$$

Therefore, along with (1) and (7), from (6) alone, we have also reconfirmed

$$
D=D_{f}+D_{p}=D_{i}+D_{P}
$$

\section{From domain integrals to maximally compact wake integrals}

The measurement area is required to be small enough for practical flow-field survey, such as a subset $W_{v}$ of $W$ where $\boldsymbol{\omega} \neq 0$. We thus transform (1) and (4) to compact vortical integrals over $W_{v} \ll W$ as much as possible, which involves only kinematic operations.

First, the profile-drag definition (1) has been recast to a moment of the Lamb vector ${ }^{29,30}$

$D_{P}=\frac{\rho}{n-1} \boldsymbol{e}_{x} \cdot \int_{W_{v}} \boldsymbol{x} \times[\boldsymbol{n} \times(\boldsymbol{u} \times \boldsymbol{\omega})] \mathrm{d} S+\frac{1}{n-1} \int_{\Sigma}(\boldsymbol{x} \times \boldsymbol{\sigma}+\boldsymbol{\tau}) \mathrm{d} S \cdot \boldsymbol{e}_{x}$, where $n=2,3$ is the spatial dimensions; $\boldsymbol{\sigma} \equiv \mu \partial \boldsymbol{\omega} / \partial \boldsymbol{n}$ and $\boldsymbol{\tau}=\mu \boldsymbol{\omega} \times \boldsymbol{n}$ are the vorticity diffusion flux and shear stress, respectively. As $\Sigma$ recedes away from the body, $\boldsymbol{\tau}$ diminishes first and then $\boldsymbol{\sigma}$ (recall that at the front and side faces of $\Sigma$, the flow is irrotational). Once $\sigma$ and $\boldsymbol{\tau}$ are negligible on $\Sigma$, by setting $\boldsymbol{x}=x \boldsymbol{e}_{x}+\boldsymbol{r}$, where $\boldsymbol{r}$ is the position vector on $W$, for $3 \mathrm{D}$ flow, (11) can be reduced to an elegant $W_{v^{-}}$ integral of a Lamb-vector moment, free from pressure measurement (Ref. 29, p. 630; see also Ref. 31),

$$
D_{P}=\frac{\rho}{2} \int_{W_{v}} \boldsymbol{r} \cdot(\boldsymbol{u} \times \boldsymbol{\omega}) \mathrm{d} S .
$$

It reveals that the physical carrier of profile drag has compact structures, which is extremely valuable for accurate calculation, measurement, and flow diagnosis. Recall $\boldsymbol{u}=\boldsymbol{U}+\boldsymbol{v}$, and $D_{P}$ can be decomposed to linear and nonlinear parts

$$
D_{P}=\frac{\rho}{2} \boldsymbol{U} \cdot \int_{W_{v}} \boldsymbol{\omega} \times \boldsymbol{r} \mathrm{d} S+\frac{\rho}{2} \int_{W_{v}} \boldsymbol{r} \cdot(\boldsymbol{v} \times \boldsymbol{\omega}) \mathrm{d} S \equiv D_{P 0}+D_{P 1} .
$$

Next, after some complex algebra detailed in Appendix A, we obtain from (7) that

$$
\begin{aligned}
D_{i}= & \frac{\rho}{2} \int_{W_{v}} \boldsymbol{\omega} \cdot \psi \mathrm{d} S+\rho \int_{W_{v}} \boldsymbol{r} \cdot(\boldsymbol{v} \times \boldsymbol{\omega}) \mathrm{d} S \\
& +\frac{\rho}{2} \frac{\mathrm{d}}{\mathrm{d} x} \int_{W}\left[\boldsymbol{e}_{x} \cdot(\boldsymbol{\psi} \times \boldsymbol{v})+2 \boldsymbol{u}^{\prime} \boldsymbol{v} \cdot \boldsymbol{r}\right] \mathrm{d} S .
\end{aligned}
$$

We call (14) the $(\boldsymbol{\omega}, \boldsymbol{\psi})$-formulation, which is evidently an extension of (3) to full nonlinearity and three dimensions. Remarkably, the second compact integral is precisely $2 D_{P 1}$ given in (13), indicating that at least in this $(\omega, \psi)$ formulation the induced drag and profile drag are inherently (but kinematically in the present context) correlated or coupled. Note, however, there is still a noncompact integral involving the $x$-derivative of disturbance variables. Technically, it is the result of integrating a 3D divergence term over $W$, say $\nabla \cdot \boldsymbol{f}$, while physically, it represents explicitly the $x$-dependency of the induced drag (and hence also the profile drag).

It is easily seen that the exact formula (14), though much less elegant than (7), includes (2) and (3) as simplified approximations. Actually, as an inviscid and linearized wake model with only $\boldsymbol{\omega}=\left(\omega_{x}, 0,0\right)$, the wake flow is governed by the inviscid Oseen equation $U \partial_{x} \boldsymbol{v}=0$, implying that the leading-order disturbances are all $x$-independent. Such a streamwise vorticity field must induce a disturbance velocity $\boldsymbol{v}=(0, v, w)$, so on each wake plane, the flow is two-dimensional with $\boldsymbol{\psi}=\psi_{x} \boldsymbol{e}_{x}$. Then in (14), there is $\boldsymbol{\omega} \cdot \boldsymbol{\psi}=\omega_{x} \psi_{x}$, noncompact $x$-derivative term vanishes, and the integral of $\boldsymbol{r} \cdot(\boldsymbol{v} \times \boldsymbol{\omega})$ is now simplified to $\boldsymbol{r} \cdot(\boldsymbol{v} \times \boldsymbol{\omega})_{2 D}$ since $u^{\prime}=0$ [see (A7)]. Therefore, when $W$ is away from the body, (14) is reduced to Maskell's formula (3). Finally, Cummings, Giles, and Shrinivas $^{11}$ have proven that for a single flat wake vortex-sheet model, (3) returns to (2).

\section{General wake-integral behaviors for attached and separated flow}

In this paper, we calculated two typical steady flows as numerical demonstration of the theory: the attached flow over an $X_{t}=1.0$ elliptic wing at Reynolds number $R e=10^{5}$ and the separated flow over a slender delta wing with sweep angle $\chi=76^{\circ}$ at Reynolds number $R e=5 \times 10^{5}$. The Reynolds numbers for both are based on the root-chord length $c$. The uniform velocity $U$ and $c$ are used to scale all data shown below. 


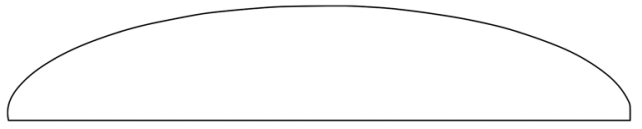

FIG. 2. $X_{t}=1.0$ elliptic wing geometry.

The $X_{t}=1.0$ elliptic wing was developed for induced drag studies and was first described in Ref. 32. The same geometry has been adopted: the NACA 0012 airfoil sectional shape, $\Lambda=7$, with the elliptic spanwise chord distribution is shown in Fig. 2. In our calculation, we take $\alpha=4^{\circ}$.

For slender delta-wing flow, in order to simplify the mesh generation, a flat-plate wing model of uniform thickness of 0.01 was used, which is shown in Fig. 3(c) and compared with the experimental models used by Earnshaw and Lawford ${ }^{33}$ and NASA [Figs. 3(a) and $3(\mathrm{~b})]$.

Computations were conducted with the steady and compressible model of ANSYS CFX. For the elliptic wing, in order to maintain the stability of vortex roll up in the wake, the $k-\epsilon$ model was applied.
For the delta wing, except the narrow regions inside the leadingedge vortices, this model flow is still laminar, and no turbulent model was applied. The vectorial streamfunction is solved by ANSYS CFX as a companion Poisson equation, for which the boundary conditions adopt the potential flow. For the compactness and neatness, the detailed validations of computations are presented in Appendix B.

In this section, most of the results are shown for varying location $x$ of the wake plane to see the $x$-dependency of drag components. The effect of shear stress $\tau$ at wake planes is found indeed very small and well negligible. In calculating all $W$-integral based force components, we found that for compact vortical integrals it suffices to take the size of $W_{v}$ as about a circle with $r=3 c$, but for the noncompact ones, the size of $W$ has to be at least a circle with $r=10 c$. The detailed discussion is presented in Appendix C.

\section{Profile drag}

We first test $D_{P}$ formula (13) in terms of the Lamb-vector moment and compare it with its primary definition (1). This is shown in Fig. 4. The maximum relative error between the two for

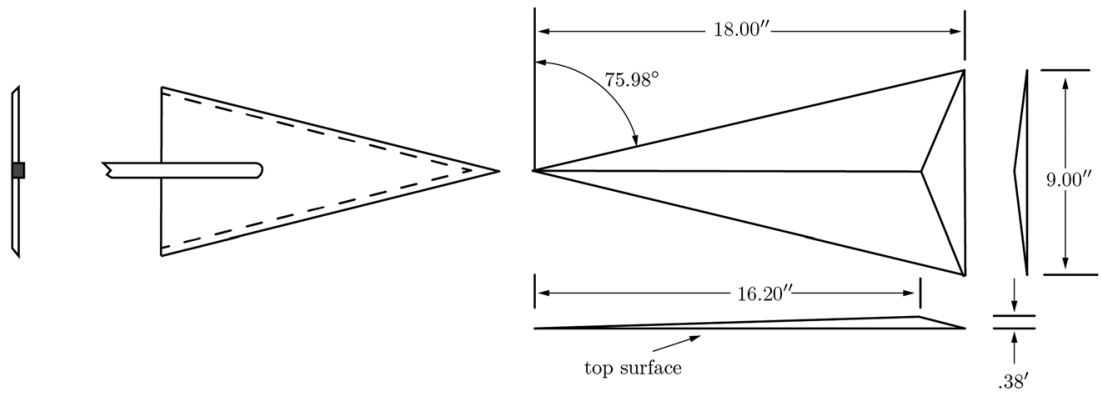

(a)

(b)

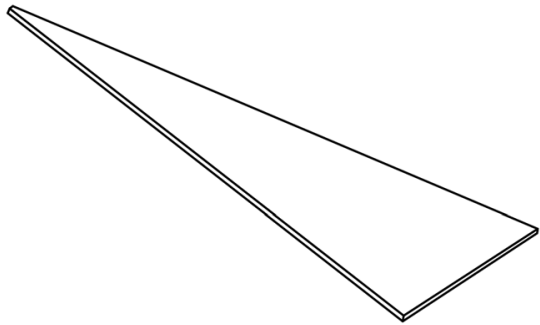

(c)

FIG. 3. Delta wing geometry. (a) Geometry used in the work of Earnshaw and Lawford; ${ }^{33}$ (b) geometry used in the work of Visser and Washburn (see Ref. 34); and (c) geometry used in the present calculation.

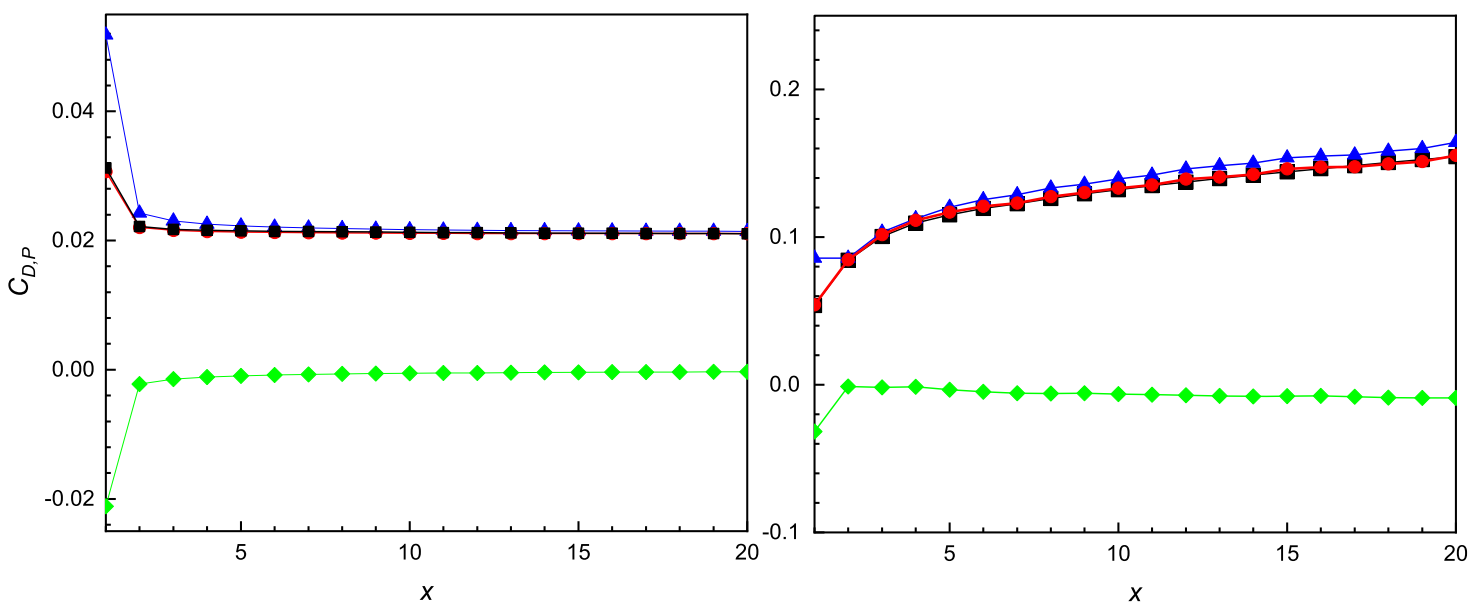

FIG. 4. The $x$-dependency of $D_{P}$ for left: elliptic wing with $\Lambda=7, R e=10^{5}$, and $\alpha=4^{\circ}$; right: delta wing with $\chi=76^{\circ}, R e=5 \times 10^{5}$, and $\alpha=20^{\circ}$. Calculated by $(1)$ (black filled square), (12) (red filled circle), and the two terms of (13): the integral of $\rho \boldsymbol{U} \cdot(\boldsymbol{\omega} \times \boldsymbol{r}) / 2$ (blue filled triangle) and $\rho \boldsymbol{r} \cdot(\boldsymbol{v} \times \boldsymbol{\omega}) / 2$ (light green filled diamond). 

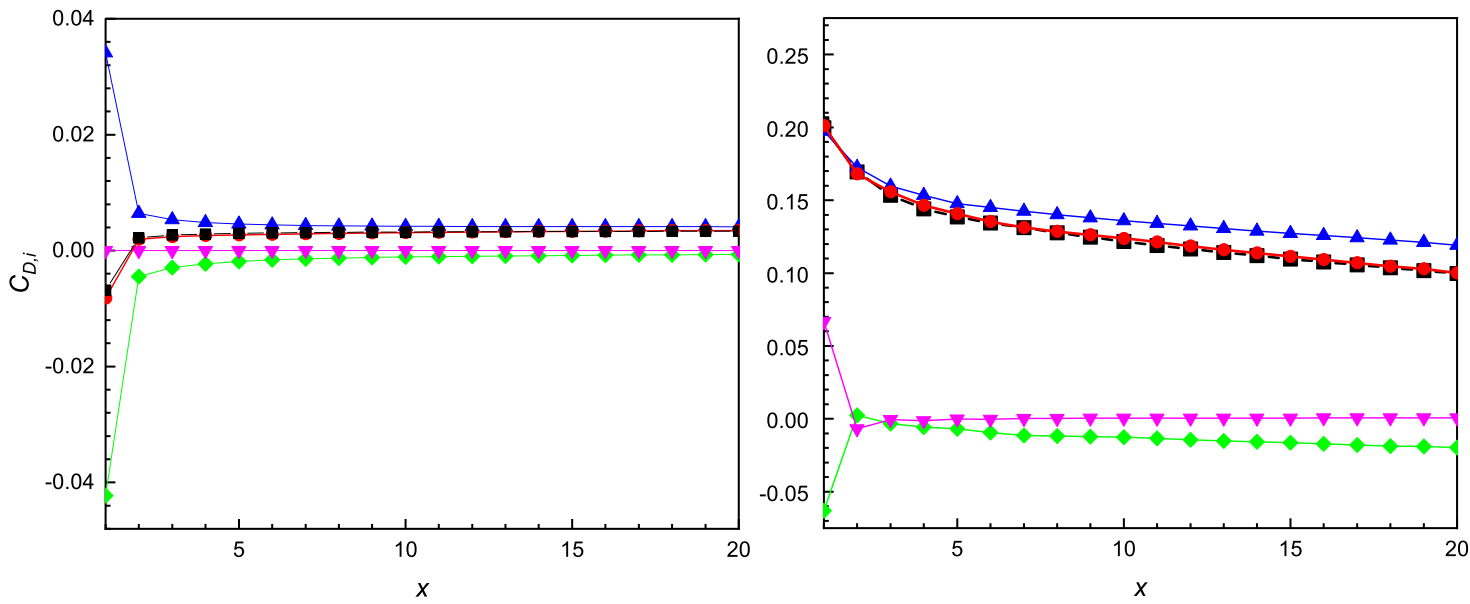

FIG. 5. The $x$-dependency of $D_{i}$ for the elliptic wing (left) and delta wing (right), with the same flow parameters as in Fig. 4. Calculated by (4) (black filled square), (14) (red filled circle), and its three terms: the integrals of $\rho \boldsymbol{\omega} \cdot \psi / 2$ (blue filled triangle), $\rho \boldsymbol{r} \cdot(\boldsymbol{v} \times \boldsymbol{\omega})$ (light green filled diamond), and $x$-derivative term (magenta filled down pointing triangle).

the whole range of $x$ is less than $2 \%$. In Fig. 4, the leading and trailing edges are at $x=0$ and $x \approx 1$, respectively. The linear and nonlinear terms of $D_{P}$ are also shown. For both elliptic and delta wings, the dominant mechanism is $D_{P 0}$, with $D_{P_{1}}$ adding some correction. The dominant term of the elliptic wing has a very large change after leaving the trailing edge and then becomes almost unchanged. However, the $D_{P 0}$ of the delta wing is almost constant near the trailing edge and then increases as $x$.

Although relatively weak, it is remarkable that the disturbance Lamb-vector moment $D_{P 1}$ always provides a thrust in the entire $x$ range. At the trailing edge of the wing, it has dramatic change, but quickly becomes nearly flat.

\section{Induced drag}

For induced drag, we first compare its velocity-based formula (4) and transformed version (14) (see Fig. 5). The two curves fit well, with the maximum relative error being less than $2 \%$ for both elliptic and delta wings, likely due to the insufficient size of $W$ for noncompact integrals and numerical errors.

Figure 5 also shows the contributions of different terms in (14). Once leaving the trailing edge, for both elliptic and delta wings the only dominant mechanism is the integral of $\rho \boldsymbol{\omega} \cdot \psi / 2$, with a minor revision due to that of $\rho \boldsymbol{r} \cdot(\boldsymbol{v} \times \boldsymbol{\omega})$. The wake patterns of the two wings are different as shown in Fig. 6 and hence so are their $D_{i}$ 's $x$ dependencies. The $D_{i}$ of the elliptic wing increases rapidly first and then becomes nearly constant when $x>10$. Physically, as the free shear layer is formed at the trailing edge where the boundary layers of both wing surfaces meet, it starts to roll up at wingtips in which $\omega_{x}$ grows rapidly first. Then, the tip vortices are solely balanced by the viscous diffusion inside their cores, so $\omega_{x}$ gently grows and tends to a constant.

In contrast, the $D_{i}$ of the delta wing keeps decreasing. The leading-edge vortices moving out of the wing will interact with

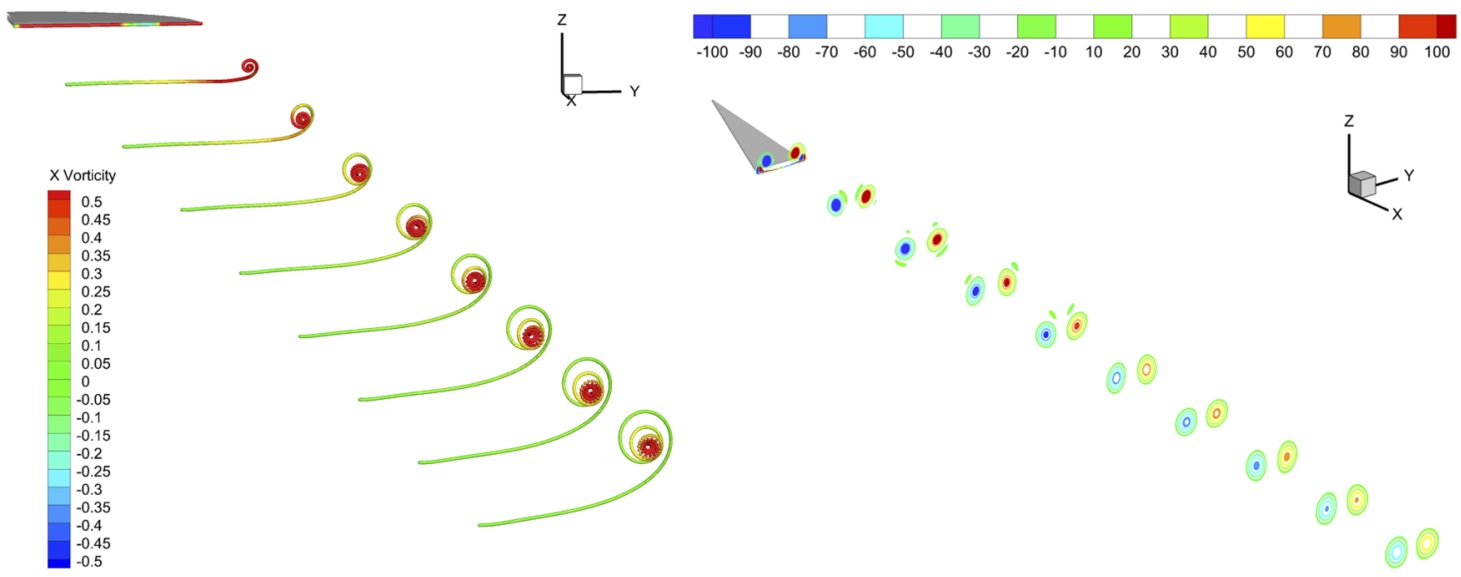

FIG. 6. The streamtracers colored by $\omega_{x}$ for the elliptic wing (left) and the contours of $\omega_{x}$ for the delta wing (right), with the same flow parameters as in Fig. 4 at different wake-plane locations. 

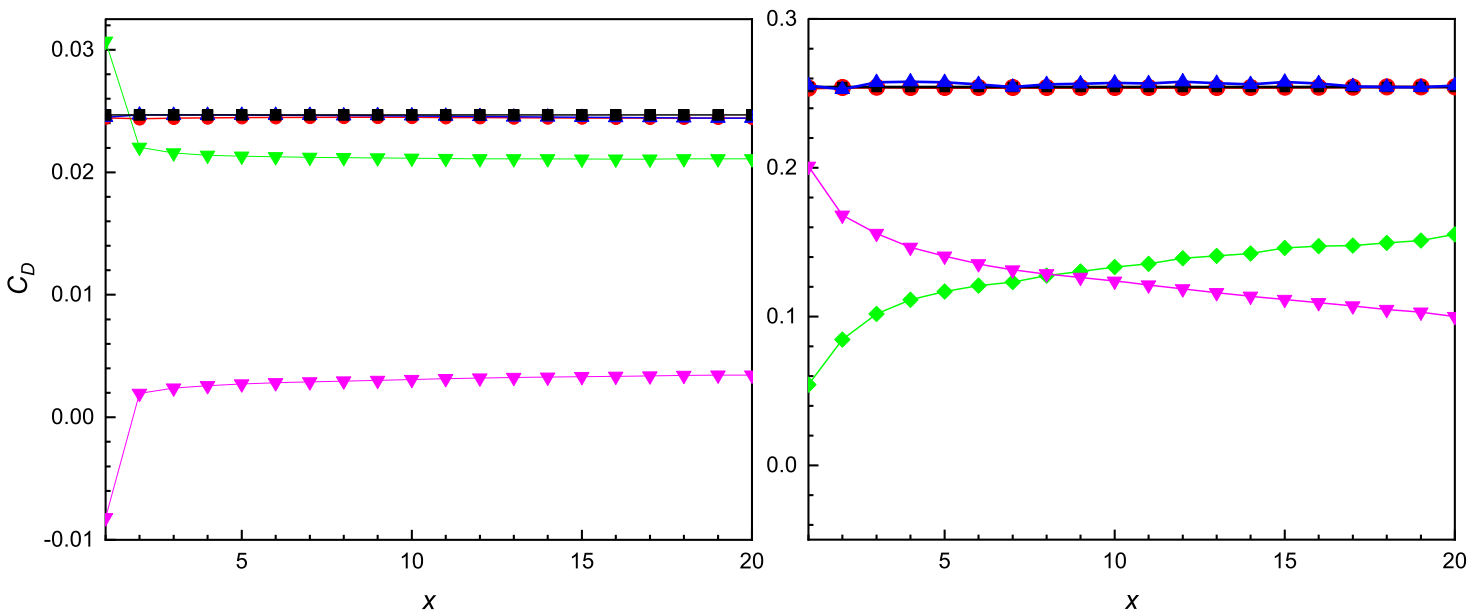

FIG. 7. The $x$-dependency of $D$ for the elliptic wing (left) and delta wing (right), with the same flow parameters as in Fig. 4. Calculated by a standard formula (black filled square), (1) + (4) (red filled circle), and (13) + (14) (blue filled triangle). $D_{P}$ and $D_{i}$ are calculated by (13) (light green filled diamond) and (14) (magenta filled down pointing triangle), respectively.

the free trailing shear layer, experiencing an intense and complicated merging process in a very short distance, and adjust to form a smooth vortical wake where $\omega_{x}$ decreases algebraically. The peak $\omega_{x}$ at the vortex center drops by a couple of times than that above the wing. However, the later evolution of this vortical wake is also mild due to viscous diffusion and dissipation.

It is worth noting that $D_{i}<0$ near the trailing edge of the elliptic wing. Unlike the delta wing, $D_{i}$ of the elliptic wing is essentially determined by the free vortex layer in the wake rather than the leading-edge vortex pair. As the free vortex layer just leaves the trailing edge, there is $u^{\prime} \approx U \gg v \approx w$ in Eq. (4). From another view, highly nonlinear flow here makes $\boldsymbol{r} \cdot(\boldsymbol{v} \times \boldsymbol{\omega})$ very large. Thus, the local $D_{i}<0$ is physical. Note that the same situation has also been found in the compressible flow (see Refs. 35 and 36).

Then, the sums (1) + (4) and (13) + (14) are used to calculate the total drag $D$ and compared with the $x$-component of the wallstress integral (8). As shown in Fig. 7, the three curves fit well, with the maximum relative errors among the three being less than $2 \%$. Both $D_{P}$ and $D_{i}$ are $x$-dependent at first. The $D_{P}$ and $D_{i}$ of the elliptic wing vary with $x$ rapidly near the trailing edge and then remain essentially unchanged. However, the $D_{P}$ of the delta wing keeps rising monotonically. On the other hand, $D_{i}$ initially accounts for the majority of $D$ and reaches a maximum near the trailing edge and then decreases monotonically, yielding the majority of $D$ to $D_{P}$ at the midwake.

\section{THE PHYSICS OF $x$-DEPENDENCY OF DRAG COMPONENTS}

The preceding theoretical and numerical results have confirmed the general $x$-dependency of $D_{i}$ and $D_{P}=D-D_{i}$ as commonly observed and exemplified that this dependency is weak for attached flow and strong for separated flow. In this section, we present a thorough analysis on the physical mechanisms responsible for this $x$-dependency, again demonstrated by numerical examples.

\section{A. Necessity of clarifying the $x$-dependency of domain/wake integrals}

In general, any force expressions by domain/wake integrals may depend on the selected domain, for which a careful check is always necessary. A classic example is the airfoil circulation $\Gamma$ in twodimensional viscous flow with $\boldsymbol{\omega}=\left(0, \omega_{y}, 0\right)$ estimated in a finite domain, for which Bryant and Williams ${ }^{37}$ found experimentally that $\Gamma$ is domain-independent. Taylor ${ }^{3}$ pointed out that this independency is ensured by the vanishing total vorticity flux across any wake plane

$$
\int_{W} u^{\prime} \omega_{y} \mathrm{~d} z=0
$$

and provided a proof of it. In retrospect, the essence of the problem is that in viscous flow, the Helmholtz decomposition of the velocity $\boldsymbol{u}=\nabla \phi+\nabla \times \boldsymbol{\psi}$ implies that there should be $\Gamma=\Gamma_{\phi}+\Gamma_{\psi}$, where $\Gamma_{\phi}$ and $\Gamma_{\psi}$ are associated with the potential flow due to the double-connectivity of the $2 \mathrm{D}$ fluid domain and the vortical wake, respectively. Condition (15) is equivalent to $\Gamma_{\psi}=0$ (Ref. 38, pp. 290-291). However, this condition does not hold in 3D flow since the fluid domain is singly connected and a vectorial $\boldsymbol{\Gamma}_{\psi}$ has an important contribution to the total lift (see Ref. 14 and Sec. 3.3.2 in Ref. 39).

Following the spirit of Taylor, ${ }^{3}$ in the rest of this section, we examine precisely why $D_{i}$ and $D_{P}$ depend on $x$, both kinematically and kinetically. This is also a necessary preparation for identifying when the possible $x$-dependency of the domain/wake-integral formulas is negligible and when it is not.

\section{B. Physical root of the $x$-dependency}

Consider the integral of any tensor field $\mathcal{F}$ over a cubic control volume $V$ with fixed front and side outer boundaries but $x$-dependent rear boundary (Fig. 8),

$$
\int_{V} \mathcal{F} \mathrm{d} V=\int_{-\infty}^{x} \mathrm{~d} x^{\prime} \int_{W(x)} \mathcal{F}\left(x^{\prime}, y^{\prime}, z^{\prime}\right) \mathrm{d} S^{\prime} .
$$




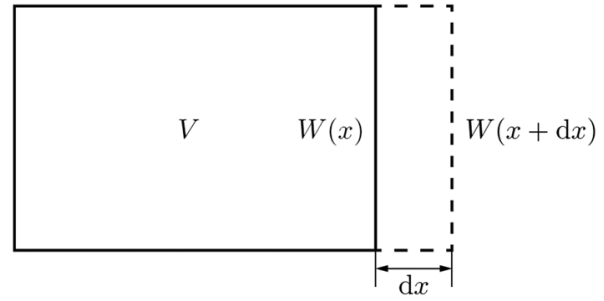

FIG. 8. Sketch of the streamwise derivative of domain integrals.

Then, as the inverse operation, it follows at once that

$$
\frac{\mathrm{d}}{\mathrm{d} x} \int_{V} \mathcal{F} \mathrm{d} V=\int_{W(x)} \mathcal{F}\left(x, y^{\prime}, z^{\prime}\right) \mathrm{d} S^{\prime}
$$

Applying (17) to (7), we obtain an exact and neat kinematic equation (tangent components of any vectors denoted by suffix $\pi$ )

$$
\frac{\mathrm{d} D_{i}}{\mathrm{~d} x}=\rho \int_{W_{v}(x)} \boldsymbol{e}_{x} \cdot(\boldsymbol{v} \times \boldsymbol{\omega}) \mathrm{d} S=\rho \int_{W_{v}(x)} \boldsymbol{e}_{x} \cdot\left(\boldsymbol{v}_{\pi} \times \boldsymbol{\omega}_{\pi}\right) \mathrm{d} S,
$$

of which the right-hand side can be easily calculated or measured. Its second expression comes from (A7). Therefore, whenever $\boldsymbol{v}_{\pi} \times \boldsymbol{\omega}_{\pi} \neq 0$ on a $W(x)$, the induced drag calculated thereon must be $x$-dependent. We stress that this should be a common case than rare because $\boldsymbol{v}_{\pi}$ must exist in the vortical wake like downwash and a nonzero total drag requires $\boldsymbol{\omega}_{\pi} \neq 0$ as explicitly implied by (22). Recall that, only if $\boldsymbol{\omega}=\left(\omega_{x}, 0,0\right), D_{i}$ would be independent of $x$, as assumed in deriving (2) and (3).

Kinetically, the best way to analyze the mechanisms behind $\mathrm{d} D_{i} / \mathrm{d} x$ is to consider $\mathrm{d} D_{P} / \mathrm{d} x=-\mathrm{d} D_{i} / \mathrm{d} x$ first. Recall $\boldsymbol{u}=\boldsymbol{U}+\boldsymbol{v}$, and from the balance between the work rate done by the body and the rate of change in kinetic energy in $V_{f}$, after some algebra one obtains

$$
\rho U D_{P}=\int_{V_{f}} \mu \omega^{2} \mathrm{~d} V-\int_{W}\left(P_{\infty}-P\right) u^{\prime} \mathrm{d} S-\mu \int_{W_{v}} \boldsymbol{e}_{x} \cdot(\boldsymbol{v} \times \boldsymbol{\omega}) \mathrm{d} S,
$$

where by the mean-value theorem there is

$$
\int_{W}\left(P_{\infty}-P\right) u^{\prime} \mathrm{d} S=\bar{u}^{\prime} \int_{W}\left(P_{\infty}-P\right) \mathrm{d} S=\overline{u^{\prime}} D_{P}
$$

Thus, we obtain

$$
\rho\left(U+\bar{u}^{\prime}\right) D_{P}=\int_{V_{f}} \mu \omega^{2} \mathrm{~d} V-\mu \int_{W_{v}} \boldsymbol{e}_{x} \cdot(\boldsymbol{v} \times \boldsymbol{\omega}) \mathrm{d} S .
$$

Then, applying (17) to (20), it follows that

$$
\begin{aligned}
\frac{\mathrm{d} D_{i}}{\mathrm{~d} x}=-\frac{\mathrm{d} D_{P}}{\mathrm{~d} x}= & -\frac{1}{\rho\left(U+\bar{u}^{\prime}\right)}\left[\int_{W_{v}(x)} \mu \omega^{2} \mathrm{~d} S\right. \\
& \left.-\mu \frac{\mathrm{d}}{\mathrm{d} x} \int_{W_{v}(x)} \boldsymbol{e}_{x} \cdot(\boldsymbol{v} \times \boldsymbol{\omega}) \mathrm{d} S\right] .
\end{aligned}
$$

Therefore, the situation with $\mathrm{d} D_{i} / \mathrm{d} x \neq 0$ happens for all viscous laminar flows at finite $R e$, all complex flows with separation, and all turbulent flow. Even at $R e \rightarrow \infty$, as the free vortex sheets roll into concentrate tip vortices with viscous subcore, ${ }^{40}$ the flow there is still dissipative.
The asymptotic behaviors of $D_{i}$ and $D_{P}$ as $x$ approaches infinity are worth examining. Recall that assuming the flow steadiness implies that we are working in a sufficiently large subzone of the free space $V_{\infty}$, say, $V_{\text {st }}$, in which the flow is treated time-independent and one can take $x \rightarrow$ " $\infty$ " in $V_{\text {st. }}$. In this limit, the first term of (20) increases monotonically, while quadratic disturbances on $W$ all die out. Then, (20) approaches the classic drag formula in terms of total dissipation, ${ }^{10,41}$ associated with $D_{i} \rightarrow 0$. This result is in full consistency with the rigorous analytical theory on force in steady compressible flow as described in Ref. 14 (see also Ref. 39). It is asserted there that for viscous flow and in the linearized far field as $x \rightarrow$ “ $\infty$," all nonlinear terms are gone so that $D_{P 1}=D_{i}=0$. What remains is the profile drag $D_{P_{0}}$ obtained by Liu et al., ${ }^{14}$ universally true for compressible flow as well,

$$
\lim _{x \rightarrow \infty^{\prime \prime}} D=D_{P}=\frac{\rho}{2} \int_{W_{v}} \boldsymbol{r} \cdot(\boldsymbol{U} \times \boldsymbol{\omega}) \mathrm{d} S .
$$

This observation further underscores the unique awkward status of the concept of induced drag: to measure it, the wake plane cannot be too far from the body, for otherwise one would simply get $D_{i}=0$; nor can it be too close to the body for otherwise $D_{i}$ would have very strong $x$-dependency.

\section{Quasiobjective vs nonobjective induced and profile drags}

It is now evident that whether there exists an interval of the wake-plane location $x$, where $\mathrm{d} D_{i} / \mathrm{d} x=-\mathrm{d} D_{P} / \mathrm{d} x \simeq 0$ within an acceptable error bound is a crucial condition to judge if the concept of induced and profile drags can be viewed "conditional" or "quasi" objective. If not, these drag components have to be viewed nonobjective. The numerical tests of Sec. II D indicate that this issue is case-relevant. We may roughly say that fully attached flows and separated flows fall into the quasiobjective and nonobjective types, respectively. The second type covers a much wider range of configurations and flow conditions than the first, from the flow over a wing-body combination to a whole aircraft. Below we continue our numerical study to exemplify these two types of wing flows.

For the elliptic wing at $\alpha=4^{\circ}$, it is described in Sec. II $D$ that $C_{D, i}$ and $C_{D, P}$ are essentially constant when $x>10$. Equation (18) asserts that this occurs when $\boldsymbol{v}_{\pi} \times \boldsymbol{\omega}_{\pi} \approx 0$ at far wake. The contours of $\rho \boldsymbol{e}_{x} \cdot\left(\boldsymbol{v}_{\pi} \times \boldsymbol{\omega}_{\pi}\right)$ at different $x$ are shown in Fig. 9 (left), which indeed decreases rapidly as $x$. Figure 9 (right) shows the $x$-dependency of $\left|\mathrm{d} C_{D, i} / \mathrm{d} x\right| \times 10^{4}$, which reduces to $O\left(10^{-1}\right)$ when $x>10$. Thus, in this midwake range, $D_{i}$ can be approximately considered as a fixed number. However, $x$ cannot be too large, for otherwise $D_{i}$ will drop to almost zero.

In contrast to the elliptic wing at $\alpha=4^{\circ}$, for the slender delta wing at $\alpha=20^{\circ}$, Fig. 10 shows the contours of $\rho \boldsymbol{e}_{x} \cdot\left(\boldsymbol{v}_{\pi} \times \boldsymbol{\omega}_{\pi}\right)$ and the $x$-dependency of $\mathrm{d} C_{D, i} / \mathrm{d} x \times 10^{4}$. This value decreases rapidly but still remains at a significant level as $x$ moves to far downstream.

Moreover, recall that the commonly used classic induced-drag formula (3) is expected to behave better at smaller $\alpha$ no matter whether the wing is slender or not, although the formula is inconsistent with (22) due to the neglect of $\boldsymbol{\omega}_{\pi}$ which is necessary for having a nonzero drag. To examine the effect of $\alpha$ on the performance of (3), Fig. 11 shows its predicted polar curves of the delta wing, calculated at $x=5$ and $x=20$, and compared with numerical result. 

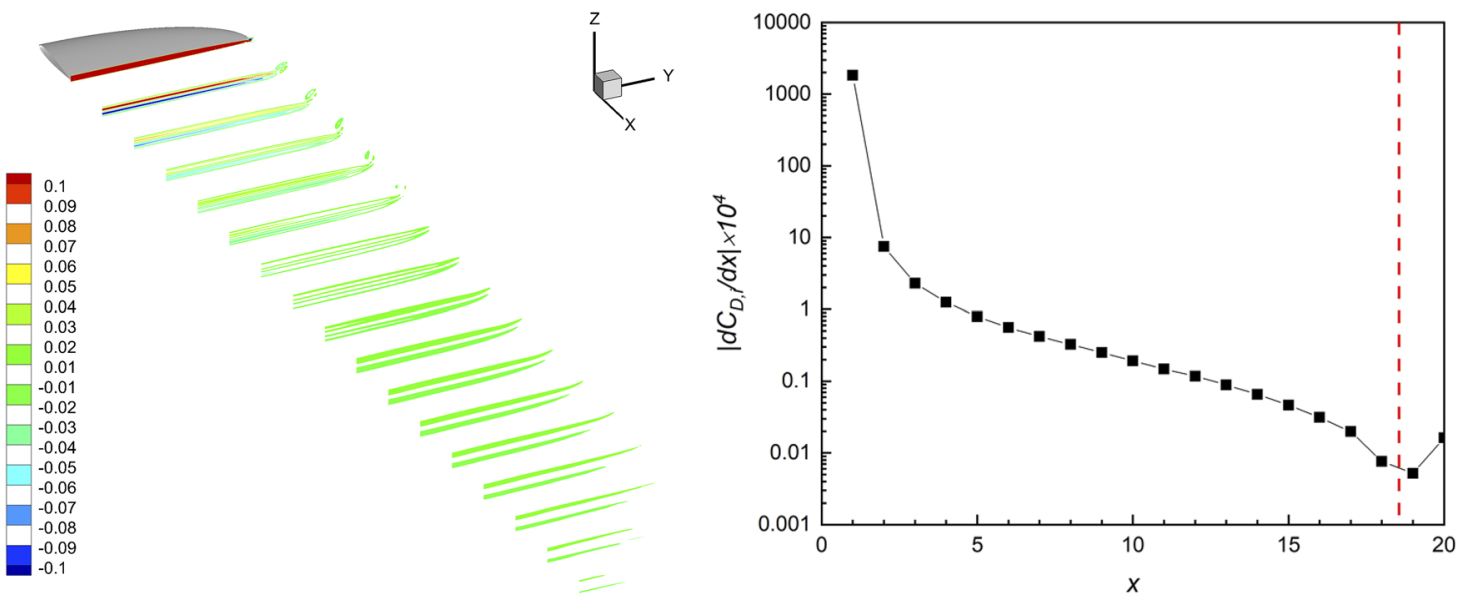

FIG. 9. (Left) The contours of $\rho \mathbf{e}_{x} \cdot\left(\boldsymbol{v}_{\pi} \times \boldsymbol{\omega}_{\pi}\right)$ of the elliptic wing at $\alpha=4^{\circ}$ and $x \in[1,20]$, normalized by $\rho U^{2} S / 2$ with $S$ being the wing area. (Right) The $x$-dependency of $\left|d C_{D, i} / d x\right| \times 10^{4}$. (The value of $d C_{D, i} / d x$ on the right-hand side of the red vertical line is negative.)
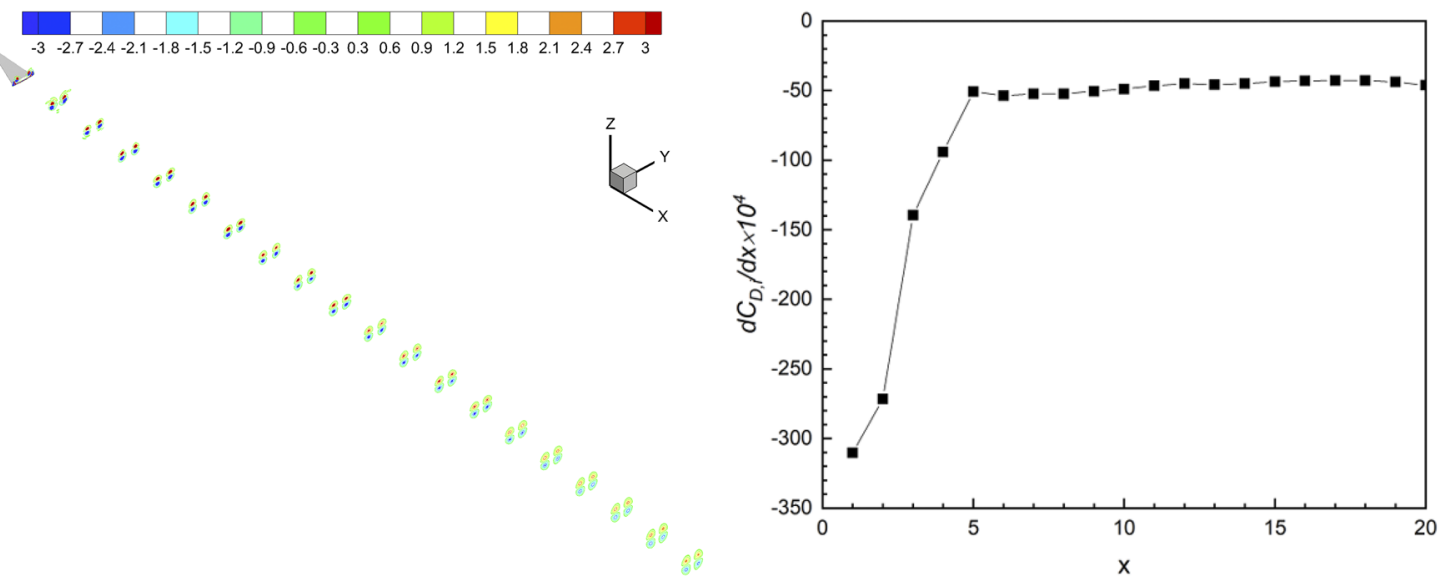

FIG. 10. (Left) The contours of $\rho \mathbf{e}_{x} \cdot\left(\boldsymbol{v}_{\pi} \times \boldsymbol{\omega}_{\pi}\right)$ of the slender delta wing at $\alpha=20^{\circ}$ and $x \in[1,20]$, normalized by $\rho U^{2} S / 2$. (Right) The $x$-dependency of $d C_{D, i} / d x \times 10^{4}$.
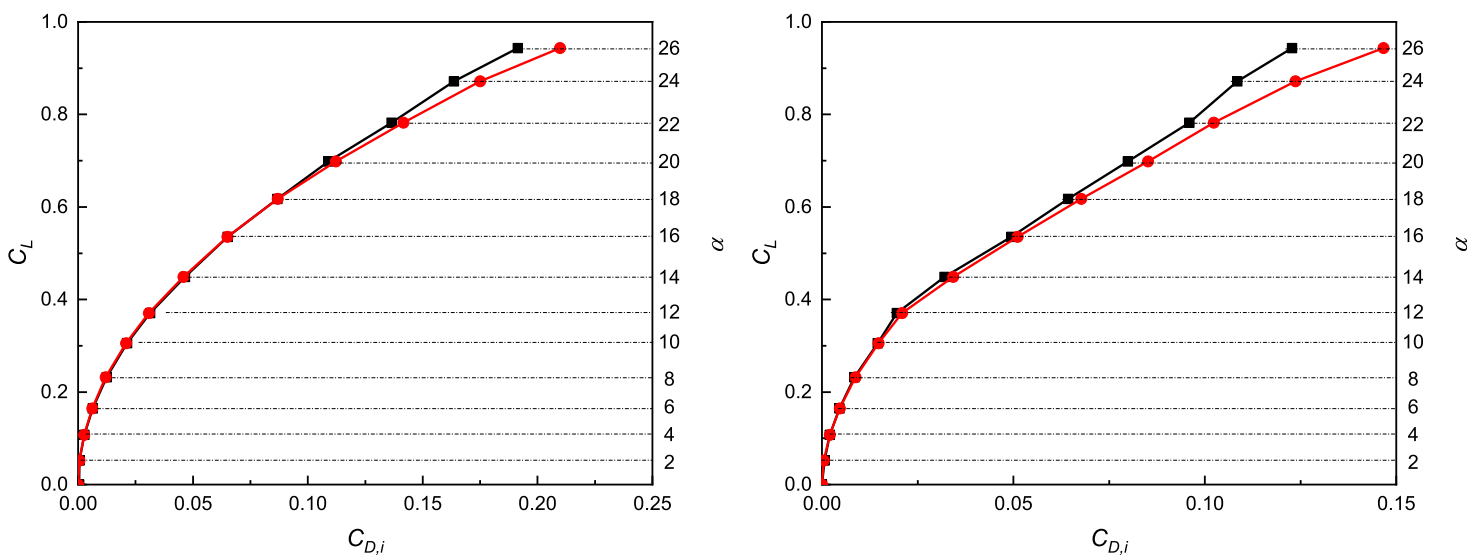

FIG. 11. The polar curve of $C_{L}$ vs induced drag $C_{D, i}$ at $x=5$ (left) and $x=20$ (right) for the delta wing with $\chi=76^{\circ}$ and $R e=5 \times 10^{5}$. Induced drag calculated by the exact wake integral (black filled square) and Maskell formula (red filled circle). 

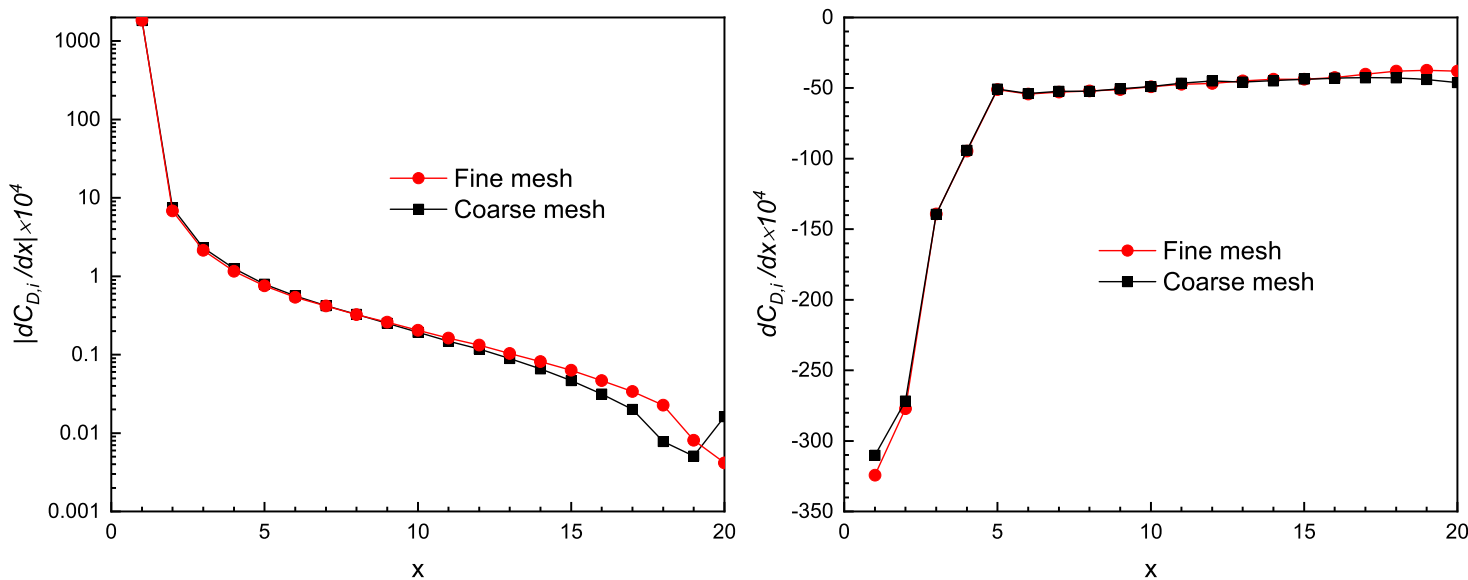

FIG. 12. The $x$-dependency of $d C_{D, i} / \mathrm{d} x \times 10^{4}$ for different grid number mesh. (Left) The elliptic wing and (right) the delta wing, with the same flow parameters as in Fig. 4 .

The two curves almost coincide when $\alpha<10^{\circ}$. Deviation begins to appear when $\alpha \geq 10^{\circ}$, and the error is too large to be acceptable when $\alpha>18^{\circ}$.

\section{Effect of numerical viscosity}

As mentioned in Sec. I, some previous studies attributed the phenomena of $D_{i}$ gradually diminishing as $x$ increases to numerical dissipation. This motivated us to distinguish the effects of physical viscosity and numerical viscosity on $\mathrm{d} D_{i} / \mathrm{d} x$. Since it is very difficult to accurately calculate the numerical viscosity, we use two indirect methods to estimate it here. The $D_{i}$ of the elliptic wing is nearly constant in the midwake, while that of the delta wing changes greatly even if it is in the far wake.

The first indirect method is to estimate the effect of grid size. The best way would be doubling the grid number in all three directions, which is, however, far beyond our computation capability. Since the streamwise evolution is the focus of the study, we just

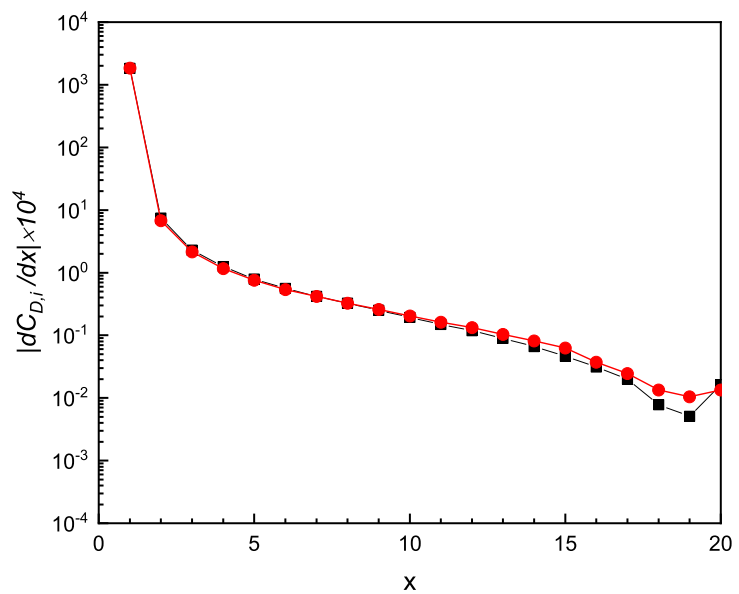

double the grid number along the streamwise direction on the previous basis and then check the results of $\mathrm{d} D_{i} / \mathrm{d} x$, which are shown in Fig. 12. The two lines fit well in most of the wake region for both the elliptic and delta wings. The numerical viscosity effect for the elliptic wing case, which is of $O\left(10^{-5}\right)$, has a visible effect when $x>10$, as shown in Fig. 12 (left). Furthermore, for the delta wing, the absolute errors of the first two points seem large, but the relative errors are within $2 \%$. The numerical viscosity has a significant effect only when $x>17$, resulting in a large deviation there.

The second indirect method is to compare (18) and (21) to test the effect of the numerical viscosity in the same case. To the end, we rewrite (21) to

$$
\begin{aligned}
\frac{\mathrm{d} D_{i}}{\mathrm{~d} x}= & -\frac{1}{\rho U}\left[\int_{W_{v}(x)} \mu \omega^{2} \mathrm{~d} S-\frac{\mathrm{d}}{\mathrm{d} x} \int_{W}\left(P_{\infty}-P\right) u^{\prime} \mathrm{d} S\right. \\
& \left.-\mu \frac{\mathrm{d}}{\mathrm{d} x} \int_{W_{v}(x)} \boldsymbol{e}_{x} \cdot(\boldsymbol{v} \times \boldsymbol{\omega}) \mathrm{d} S\right] .
\end{aligned}
$$

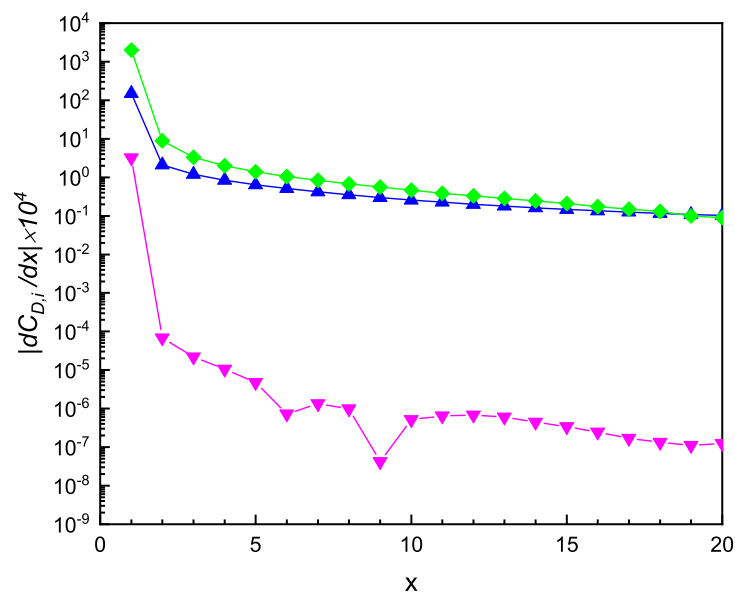

FIG. 13. The $x$-dependency of $\left|d C_{D, j} / \mathrm{d} x\right| \times 10^{4}$ for the elliptic wing, with $\Lambda=7, R e=10^{5}$, and $\alpha=4^{\circ}$. (Left) Calculated by (18) (black filled square) and (21) (red filled circle) (Right) Calculated by three terms in (23): the integrals of $\left|\left(\mu+\mu_{t}\right) \omega^{2} / \rho U\right|$ (blue filled triangle), $\left|\mathrm{d}\left[\left(P_{\infty}-P\right) u^{\prime} \mid \rho U\right] / \mathrm{d} x\right|$ (light green filled triangle), and $\left(\mu+\mu_{t}\right) \mid \mathrm{d}\left[\boldsymbol{e}_{x} \cdot(\boldsymbol{v} \times \boldsymbol{\omega}) \mid\right.$ $\rho U] / d x \mid$ (magenta filled down pointing triangle). 

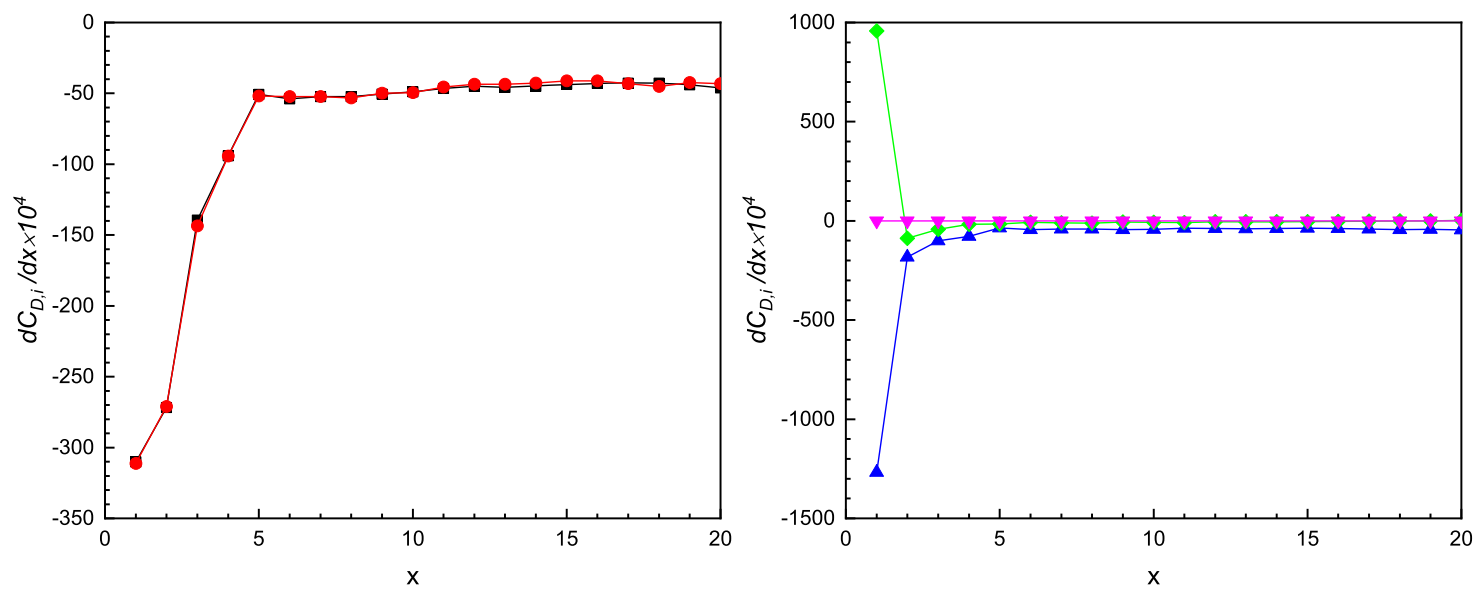

FIG. 14. The $x$-dependency of $d C_{D, i} / \mathrm{d} x \times 10^{4}$ for the delta wing, with $\chi=76^{\circ}, R e=5 \times 10^{5}$, and $\alpha=20^{\circ}$. (Left) Calculated by (18) (black filled square) and (21) (red filled circle). (Right) Calculated by three terms in (23): the integrals of $-\mu \omega^{2} / \rho U$ (blue filled triangle), $\mathrm{d}\left[\left(P_{\infty}-P\right) u^{\prime} / \rho U\right] / \mathrm{d} x$ (light green filled diamond), and $\mu \mathrm{d}\left[\boldsymbol{e}_{x} \cdot(\boldsymbol{v} \times \boldsymbol{\omega}) / \rho U\right] / \mathrm{d} x$ (magenta filled down point triangle).

As shown in Fig. 13 (left) and Fig. 14 (left), two lines almost coincide for the elliptic and delta wings. Their difference can be regarded as the effect of numerical viscosity. Obviously, most of the effects come from the molecular viscosity $\mu$ and eddy viscosity $\mu_{t}$, where the latter exists in the elliptic wing case. Detailed physical sources of streamwise evolution are shown in Fig. 13 (right) and Fig. 14 (right). The $\mathrm{d}\left[\left(P_{\infty}-P\right) u^{\prime} / \rho U\right] / \mathrm{d} x$ (light green filled diamond) and physical dissipation $-\tilde{\mu} \omega^{2} / \rho U$ (blue filled triangle) are the major sources, where $\tilde{\mu}=\mu$ or $\left(\mu+\mu_{t}\right)$ for the laminar or turbulent flow, respectively. The $\tilde{\mu} \mathrm{d}\left[\boldsymbol{e}_{x} \cdot(\boldsymbol{v} \times \boldsymbol{\omega}) / \rho U\right] / \mathrm{d} x$ is nearly zero for both cases that can be well ignored. Since the flow field near the trailing edge is highly nonlinear, $\mathrm{d}\left[\left(P_{\infty}-P\right) u^{\prime} / \rho U\right] / \mathrm{d} x$ and physical dissipation are large. Then, for the elliptic wing, $\left|\tilde{\mu} \omega^{2} / \rho U\right|$ decreases fast at first and then becomes nearly stable as $x$ increases, while $\left|\mathrm{d}\left[\left(P_{\infty}-P\right) u^{\prime} / \rho U\right] / \mathrm{d} x\right|$ continues to decrease to be smaller than the former when $x>18$. For the delta wing, the amplitude of $\mathrm{d}\left[\left(P_{\infty}-P\right) u^{\prime} / \rho U\right] / \mathrm{d} x$ decreases very quickly as $x$ increases, of which most comes from the viscous dissipation term when $x>5$. Both also confirm that $D_{i}=0$ when $x \rightarrow$ " $\infty$ " is due to physical dissipation.

In summary, the physical mechanism and vortical structures responsible for the true $x$-dependency of these drag components are well clarified by a combined use of theoretical and numerical approaches.

\section{COMPACT MIDWAKE APPROXIMATION OF INDUCED DRAG}

In engineering practice, one wishes to find the $x$-locations in the viscous wake where $\mathrm{d} D_{i} / \mathrm{d} x \simeq 0$ so that a flow survey over a single vortical $W_{v}$ can yield the induced drag within acceptable error. As described earlier, the only choice of proper $x$ is the weakly nonlinear zone in between, which we call the midwake.

On such a midwake plane $W$, the core nonlinear effect, namely, the disturbance Lamb vector $\boldsymbol{v} \times \boldsymbol{\omega}$, should be reserved as inherently required by the nature of induced drag. However, due to the smallness of $x$-derivative terms, not only the viscous stress on $W$ but also those explicitly $x$-dependent noncompact integrals in (14) can be dropped. This leads an approximation

$$
D_{i} \simeq \frac{\rho}{2} \int_{W_{v}} \boldsymbol{\omega} \cdot \psi \mathrm{d} S+\rho \int_{W_{v}} \boldsymbol{r} \cdot(\boldsymbol{v} \times \boldsymbol{\omega}) \mathrm{d} S .
$$

Moreover, technically, the $3 \mathrm{D}$ stream-function $\psi$ is still troublesome as its calculation involves the whole flow-field information. To remove this difficulty, notice that $\partial_{x} \ll\left(\partial_{y}, \partial_{z}\right)$ in the midwake, and the gradient operator $\nabla$ can be simplified to $\nabla_{\pi}$ that only involves tangent derivatives on a single wake plane. Namely, we define a vector $\tilde{\psi}$ by

$$
\nabla_{\pi}^{2} \tilde{\psi} \simeq-\boldsymbol{\omega} \text { such that } \boldsymbol{\omega} \cdot \boldsymbol{\psi} \simeq \boldsymbol{\omega} \cdot \tilde{\boldsymbol{\psi}}
$$

This leads to a more convenient compact midwake approximation

$$
D_{i} \simeq \frac{\rho}{2} \int_{W_{v}} \boldsymbol{\omega} \cdot \tilde{\psi} \mathrm{d} S+\rho \int_{W_{v}} \boldsymbol{r} \cdot(\boldsymbol{v} \times \boldsymbol{\omega}) \mathrm{d} S .
$$

We now examine the prediction of (25), using the same ellipticwing and slender delta-wing flows as the numerical test bed, for which the midwake region may be roughly identified as $x \in(3,20)$.

The $x$-evolution of the induced-drag calculated by formulas (14), (25), (2), and (3) is shown in Fig. 15. Because to obtain the Fourier coefficients of the lift distribution for the delta wing is difficult and beyond our scope, Prandtl's formula (2) is only used for the elliptic wing with $\delta=0$ and yields a straight line. Maskell's formula (3) behaves much better but has a large error in the near wake region $(x=2)$ as expected. For the elliptic wing, its error becomes smaller as $x$ increases and eventually overlaps when $x>18$. For the delta wing, surprisingly, its error is very small when $2 \leq x \leq 4$. A careful examination of the flow field reveals that in this region $\omega_{x}$ is an order of magnitude larger than $\boldsymbol{\omega}_{\pi}$, which also leads the value of $\rho \int_{W_{v}} \boldsymbol{r} \cdot(\boldsymbol{v} \times \boldsymbol{\omega})$ here nearly zero. But for $x>4, \omega_{x}$ first decreases rapidly and then gradually stabilizes, while $\boldsymbol{\omega}_{\pi}$ does not decrease so significantly and hence cannot be ignored. Consequently, the error of (3) gradually increases, reaching $9 \%$ when $x=20$. As shown in 

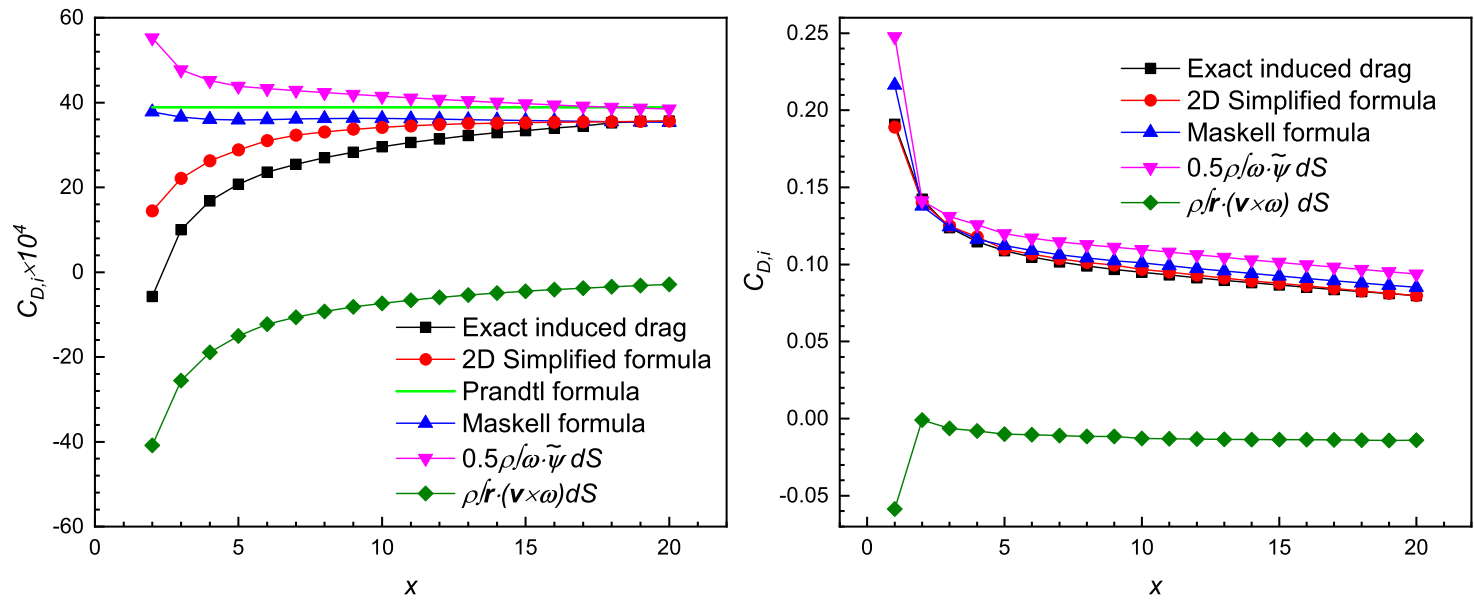

FIG. 15. The $x$-dependency of induced drag for the elliptic wing (left) and delta wing (right), with the same flow parameters as in Fig. 4. Induced drag calculated by (14) (black filled square), (25) (red filled circle), Maskell formula (3) (blue filled triangle), and Prandtl formula (2) (light green solid line). Two terms in (25), $0.5 \rho \int_{W_{v}} \boldsymbol{\omega} \cdot \tilde{\psi} \mathrm{d} S$ (magenta filled down point triangle) and $\rho \int_{W_{v}} \boldsymbol{r} \cdot(\boldsymbol{v} \times \boldsymbol{\omega}) \mathrm{d} S$ (dark green filled diamond), are also plotted.

Fig. 15, although the trends of $0.5 \rho \int_{W_{v}} \boldsymbol{\omega} \cdot \tilde{\psi} \mathrm{d} S$ and Maskell's formula (3) are consistent, the former has greater deviation from the exact value than the latter. It is the $\boldsymbol{r} \cdot(\boldsymbol{v} \times \boldsymbol{\omega})$, which is the core nonlinear effect of induced drag, which corrects this deviation. Therefore, compared with (2) and (3), (25) can significantly improve the accuracy of induced drag prediction over the whole $x$ range.

No wonder, aerodynamicists prefer the Maskell formula because $D_{i}$ is almost invariant for the elliptic wing as $x$ increases. However, taking $D_{P}$ 's formula (1) into consideration at the same time, when $x<10$ for the elliptic wing, we find $D \neq D_{i}+D_{P}$ if $D_{i}$ is obtained by Eq. (3). Furthermore, for the flow around a generic configuration, such as a wing-body configuration with nacelle/pylon/horizontal-tail (e.g., Ref. 42) or a full aircraft, the flow field is inevitably separated, for which the streamwise evolution of $D_{i}$ should depend on $x$ appreciably. In the delta-wing wake, although the qualitative trend of Eq. (3) is correct in the near field due to special reasons, the quantitative prediction in the midwake is poor. In contrast, the midwake approximation faithfully reflects the trend of induced drag and is valid as long as $x>2$.

It should be born in mind that, as indicated by (22), assuming $\boldsymbol{\omega}=\left(\omega_{x}, 0,0\right)$ as in (3) conflicts directly the very existence of the drag that in viscous flow is always associated with nonzero $\omega_{\pi}$. Only if $D=0$, could the wake vorticity have just one $x$-component and be $x$-independent. This nonphysical oversimplification of (3) also explains why it cannot predict the correct $x$-variation trend in the near field and perhaps is one of the reasons that Spalart ${ }^{9}$ uses coordinates $\left(x^{\prime}, y, z^{\prime}\right)$ with a downward inclined $x^{\prime}$ axis. In summary, the Trefftz plane, which is the basis of Maskell's formula, is physically paradoxical in viscous flow, while a wake plane that satisfies midwake approximation (24) and (25) does exist in large $x$ intervals.
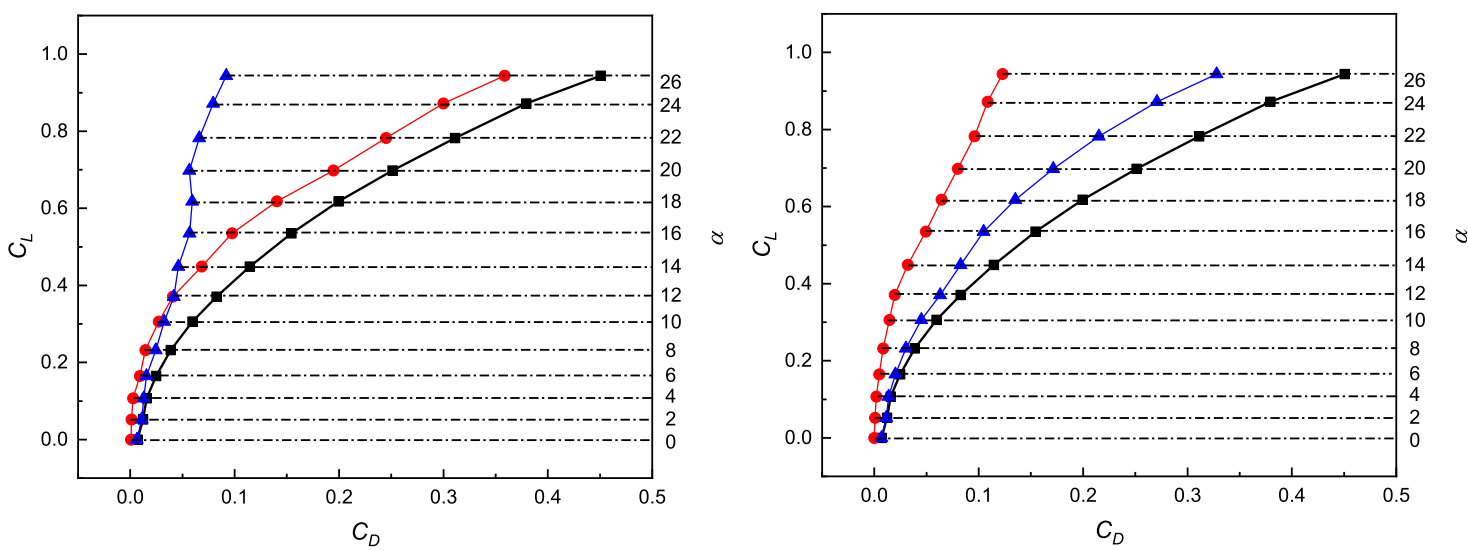

FIG. 16. The polar curve of $C_{L}$ vs drag $C_{D}$ and its components for the delta wing with $\chi=76^{\circ}, R e=5 \times 10^{5}$. (Left) $x$ at the trailing edge. (Right) $x=20$. Drag calculated by a standard formula (black filled square) and two terms: induced drag (red filled circle) and profile drag (blue filled triangle). 
Before ending this section, we make another important observation on which drag, $D_{i}$ or $D_{P}$, should be the main target of dragreduction studies. Figure 7 (left) has shown that for the elliptic wing at small $\alpha, D_{P}$ keeps larger than $D_{i}$ by about an order of magnitude, and for the slender delta wing at large $\alpha, D_{i}$ decays to a level smaller than $D_{P}$ after $x>9$. While the relative importance of $D_{i}$ and $D_{P}$ may depend strongly on the Reynolds number and the present numerical example is not conclusive, the relative importance can be judged more definitely from the polar curves. Figure 16 shows the partition of total drag $D$ into $D_{i}$ and $D_{P}$ vs $C_{L}$ for the slender delta wing. Near the trailing edge, $D_{i}$ dominates $D$, but at $x=20, D_{P}$ becomes about three times of $D_{i}$. This being the case, then, the major target of dragreduction studies for at least separated flow should be shifted from induced drag to profile drag. With the elegant formula (12) at hand, no matter which component is larger, this should be an even easier task and with a more accurate result than sticking to the induced drag.

\section{CONCLUSIONS AND DISCUSSIONS}

This paper grows naturally from the well-established viscous vortex-force theory, which has been extensively verified and exemplified by numerical studies of highly nonlinear flows.

1. A general and exact theory on the profile and induced drags for 3D incompressible, viscous, and steady flow over a generic body is presented, in terms of domain/wake integrals. The transformation of domain integrals to wake-plane integrals can significantly simplify the flow-field survey technique, but in the formula for $D_{i}$, there still remain some extra noncompact and explicitly $x$-dependent terms. The exact wake integrals found by the present theory are applicable to the entire wake region and have full consistency with the existing drag-breakdown theories.

2. The precise physical root of the observed $x$-dependency of $D_{i}$ and $D_{P}$ is identified. The result not only confirms that, as a generic rule, the drag components expressed by wake integrals must depend on the choice of wake-plane streamwise location but also enables observing specific dynamic processes of flow structures responsible for the streamwise evolution of these force components. The innovative kinematic formula (18) for the $x$-derivative of induced drag, $\mathrm{d} D_{i} / \mathrm{d} x$, reveals that it is exactly determined by a $W_{v}$-integral of the disturbance Lamb vector $\boldsymbol{v} \times \boldsymbol{\omega}$, while the kinetic formula (21) for $\mathrm{d} D_{i} / \mathrm{d} x=-\mathrm{d} D_{P} / \mathrm{d} x$ reveals that assuming $x$-independence of $D_{i}$ implies neglecting the dissipation over the wake-plane. In the linear and viscous far wake, $D_{i}$ vanishes and $D_{P}$ becomes the total drag $D$. The numerical examples indicate that the concept of induced and profile drags as fixed numbers can at most be approximately true for attached flows, and these drags must strongly depend on $x$ for more complex separated flows.

3. A new compact midwake approximation of the exact $D_{i}$ formula is proposed, applicable if the wake plane locates at a weakly nonlinear midwake region where the $x$-dependency of $D_{i}$ is weak. This new formula retains the compact nonlinear Lamb-vector integrals that is the key mechanism for the induced drag and hence significantly improves the prediction of classic induced-drag formulas which were also designed to be used in the same region. The new formula may serve to evaluate the overall force performance in preliminary configuration design, but with a warning that the induced drag there could already become smaller than the profile drag.

The above findings motivated us to ponder the entire subject of drag breakdown. Some of our thoughts are presented here as a final discussion.

1. A comparison between $D_{i}$ and $D_{P}$.

At least one of $D_{i}$ and $D_{P}$ have to be defined theoretically and both suffer from the $x$-dependency problem, but their behaviors are drastically different. The primary definition of $D_{P}$ is the integrated total pressure deficit in the wake, with the integrand being the gradient of a scalar. Thus, $D_{P}$ can well be cast to an elegant and compact $W_{v}$-integral of a Lamb-vector moment. Moreover, it contains linear and nonlinear parts, and the linear part is large enough to ensure the value of $D_{P}$ be basically captured.

In contrast, the primary definition of $D_{i}$ has to be a domain integral of solely nonlinear disturbance Lamb vector in a $3 \mathrm{D}$ flow field (which is homologous to the nonlinear part of the lift). Consequently, first, the exact $D_{i}$ formula cannot be a fully compact wake integral. Second, its intrinsic nonlinearity makes it destined to have some unique properties: it is hard to be accurately captured; it should be best surveyed in the nearwake region; but one's wish that it should be a fixed number can only be realized for simple attached flow and surveyed in a wake region far from the body; yet, it must disappear as $x \rightarrow \infty$. These inherently conflicting needs are the very cause of the theoretical and practical difficulties in its wake-plane survey and has been making the induced drag the major shortcoming of the whole field of drag prediction.

2. On the relaxation of the concepts of induced and profile drags.

The unique status of the very concept of induced drag, and the inevitable $x$-dependency of both $D_{i}$ and $D_{P}$, is worth deep reflecting. Recall that the induced drag was introduced by Prandtl as a unique drag in inviscid flow, and in the specific simplification behind (2), it does appear as a fixed number. Once the viscosity entered into play, however, the $x$ dependency $D_{i}$ and $D_{P}$ appeared at once as already implied by Maskell's formula (3). True, the $x$-independency can be approximately recovered for simple attached flows at a carefully chosen wake-plane location, which is why the conventional wish that $D_{i}$ and $D_{P}$ be fixed numbers has been proved useful in routine aerodynamic configuration designs. However, in developing innovative configurations with complex separated flows, we see no reason to continuously stick to that wish. Instead, we propose to totally abandon the "fixednumber wish" but admit the $x$-dependency of $D_{i}$ and $D_{P}$ as the nature tells.

Then, recall (7) and (12), the two drag components as well as the lift are just difference faces of the same Lamb-vector field and its moments generated by the body's steady motion, and these faces are inherently coupled. Hence, they should best be studied in the near wake to avoid the rich information be contaminated by physical and numerical dissipation. Realizing this truth could lead to an innovative methodology in drag decomposition and reduction, characterized by shifting one's 
attention from induced and profile drags as numbers to the Lamb-vector field as a function of spatial location. This possible new methodology is under investigation, of which the result will be reported elsewhere as a continuation of the present paper.

\section{ACKNOWLEDGMENTS}

This work was supported by the National Natural Science Foundation of China (Grant No. 11472016). The authors are very grateful to Professor Xijin Zhang and Dr. Miao Zhang for motivating this research project and Professor Weidong Su, Dr. Rikui Zhang, Dr. Zhoufu Li, Dr. Zhansen Qian, Dr. Qing Shen, and Dr. Peng Bai for valuable discussions. The authors also thank the referees for their constructive comments, which significantly improved this paper.

\section{APPENDIX A: IDENTITIES AND DETAILED DERIVATION}

\section{Derivative-moment transformations}

Let $f$ be any smooth vector field and $\phi$ be any smooth scalar field, $x$ be the position vector with arbitrary origin, and $n=2,3$ be the spatial dimensions. Then, ${ }^{29}$

$$
\begin{gathered}
\int_{\mathcal{D}} \boldsymbol{f} \mathrm{d} V=-\int_{\mathcal{D}} \boldsymbol{x}(\nabla \cdot \boldsymbol{f}) \mathrm{d} V+\int_{\partial \mathcal{D}} \boldsymbol{x}(\boldsymbol{n} \cdot \boldsymbol{f}) \mathrm{d} S \\
\int_{S} \phi \boldsymbol{n} \mathrm{d} S=-\frac{1}{n-1} \int_{S} \boldsymbol{x} \times(\boldsymbol{n} \times \nabla \phi) \mathrm{d} S+\frac{1}{n-1} \oint_{\partial S} \phi \boldsymbol{x} \times \mathrm{d} \boldsymbol{x} .
\end{gathered}
$$

\section{Identities involving the vorticity and Lamb vector}

Let $\boldsymbol{v}=\left(u^{\prime}, v, w\right)$ be the disturbance velocity, $\boldsymbol{\omega}=\nabla \times \boldsymbol{v}$ $=-\nabla^{2} \psi, k \equiv|\boldsymbol{v}|^{2} / 2$, and $\mathbf{I}$ be the unit tensor. Assume $\nabla \cdot \boldsymbol{v}=0$. Then,

$$
\begin{aligned}
2 k & =\nabla \cdot(\boldsymbol{\psi} \times \boldsymbol{v})+\boldsymbol{\omega} \cdot \boldsymbol{\psi}, \\
\boldsymbol{v} \times \boldsymbol{\omega} & =\nabla \cdot(k \mathbf{I}-\boldsymbol{v} \boldsymbol{v}), \\
\boldsymbol{x} \cdot(\boldsymbol{v} \times \boldsymbol{\omega}) & =\nabla \cdot(k \boldsymbol{x}-\boldsymbol{v} \boldsymbol{v} \cdot \boldsymbol{x})+(n-2) k .
\end{aligned}
$$

\section{Notations for disturbance Lamb vector}

For truly 2D flow on a $(y, z)$-plane, $\boldsymbol{\omega}=\omega_{x} \boldsymbol{e}_{x}$ and $\boldsymbol{\omega}_{\pi}=0$. Here, we denote tangent components of any vectors by suffix $\pi$. Thus, we may write

where

$$
\boldsymbol{v} \times \boldsymbol{\omega}=(\boldsymbol{v} \times \boldsymbol{\omega})_{2 D}+(\boldsymbol{v} \times \boldsymbol{\omega})_{3 D}
$$

$$
(\boldsymbol{v} \times \boldsymbol{\omega})_{2 D}=\boldsymbol{v}_{\pi} \times \omega_{x} \boldsymbol{e}_{x}, \quad(\boldsymbol{v} \times \boldsymbol{\omega})_{3 D}=\boldsymbol{e}_{x} u^{\prime} \times \boldsymbol{\omega}_{\pi}+\boldsymbol{v}_{\pi} \times \boldsymbol{\omega}_{\pi} .
$$

Then, set $\boldsymbol{x}=x \boldsymbol{e}_{x}+\boldsymbol{r}, \boldsymbol{r}=y \boldsymbol{e}_{y}+z \boldsymbol{e}_{z}$, there is

$$
\boldsymbol{x} \cdot(\boldsymbol{v} \times \boldsymbol{\omega})=\boldsymbol{r} \cdot(\boldsymbol{v} \times \boldsymbol{\omega})_{2 D}+\boldsymbol{r} \cdot(\boldsymbol{v} \times \boldsymbol{\omega})_{3 D}+x \boldsymbol{e}_{x} \cdot(\boldsymbol{v} \times \boldsymbol{\omega})_{3 D} .
$$

\section{Derivation of induced drag formula (14)}

Again denote tangent components of any vectors by suffix $\pi$, so for velocity and vorticity we set

$$
\boldsymbol{v}=\boldsymbol{v}_{\pi}+u^{\prime} \boldsymbol{e}_{x}, \boldsymbol{\omega}=\boldsymbol{\omega}_{\pi}+\boldsymbol{e}_{x} \omega_{x},
$$

where

$$
\boldsymbol{\omega}_{\pi}=\boldsymbol{e}_{x} \times\left(\partial_{x} \boldsymbol{v}_{\pi}-\nabla_{\pi} u^{\prime}\right), \quad \boldsymbol{e}_{x} \omega_{x}=\nabla_{\pi} \times \boldsymbol{v}_{\pi}
$$

We shall deal with different components of the disturbance Lamb vector $\boldsymbol{v} \times \boldsymbol{\omega}$ and its scalar moments with $\boldsymbol{x}$ and $\boldsymbol{r}$, of which some basic results are listed above.

To cast the apparently neat formula (4) for induced drag to compact integrals as much as possible, consider the wake integral of $-u^{\prime 2}$ first. In three dimensions, identity (A5) has a corollary specified to the integrals over volume $V$ and wake-plane $W$,

$$
\boldsymbol{r} \cdot(\boldsymbol{v} \times \boldsymbol{\omega})=\nabla \cdot(k \boldsymbol{r}-\boldsymbol{v} \boldsymbol{v} \cdot \boldsymbol{r})-u^{\prime 2} .
$$

Note that the $W$-integral of the divergence of any vector $f$ that is negligible at $\partial W$ can be reduced to

$$
\int_{W} \nabla \cdot \boldsymbol{f}(x, y, z) \mathrm{d} S=\frac{\mathrm{d}}{\mathrm{d} x} \int_{W} f_{x} \mathrm{~d} S .
$$

Thus, for the integral of (A10), we set $\boldsymbol{f}=k \boldsymbol{r}-\boldsymbol{v} \boldsymbol{v} \cdot \boldsymbol{r}$ such that $f_{x}=-u^{\prime} \boldsymbol{v} \cdot \boldsymbol{r}$ and find

$$
-\int_{W} u^{\prime 2} \mathrm{~d} S=\int_{W_{v}} \boldsymbol{r} \cdot(\boldsymbol{v} \times \boldsymbol{\omega}) \mathrm{d} S+\frac{\mathrm{d}}{\mathrm{d} x} \int_{W} u^{\prime} \boldsymbol{v} \cdot \boldsymbol{r} \mathrm{d} S .
$$

For the remaining integral of disturbance kinetic energy $k$ in (4), Lamb ${ }^{43}$ has offered two formulas to express it by vorticity. The first uses identity (A3) due to Helmholtz. ${ }^{44}$ Along with (A12), we obtain (14).

It may be mentioned that if we substitute the second formula of Lamb ${ }^{43}$ for kinetic energy in terms of vorticity (A5) to (4) and calculate $D_{i}$, then after some algebra we would be led to nowhere else but a remarkable formula ${ }^{18}$ on $\mathrm{d} D_{i} / \mathrm{d} x$ as have been neatly derived in Sec. III B.

\section{APPENDIX B: NUMERICAL VALIDATIONS}

For slender delta-wing flow, our numerical results will be compared with the lift and drag data measured by Earnshaw and Lawford $^{33}$ at a wide range of angles of attack $\alpha$ (EL experiment for short), and the lift and drag at $\alpha=20^{\circ}$ as well as the wake survey measured by Visser and Washburn at NASA Langely Research Center in 1995 (unpublished, referred to as NASA experiment), of which the data were analyzed by $\mathrm{Wu}$, Ondrusek, and $\mathrm{Wu}^{34}$ (but with incorrect drag breakdown). Yang et al. ${ }^{31}$ made a CFD analysis of the same flow but focused on the physical mechanism of lift. The comparison of computed $C_{L}$ and $C_{D}$ with EL and NASA experimental data indicates

TABLE I. Validation of grid. (a) Elliptic wing with $\Lambda=7, R e=10^{5}$, and $\alpha=4^{\circ}$; (b) delta wing with $\chi=76^{\circ}, R e=5 \times 10^{5}$, and $\alpha=20^{\circ}$.

\begin{tabular}{lccc}
\hline \hline Grid numbers (million) & & $C_{L}$ & $C_{D}$ \\
\hline & (a) & & \\
\hline 6.5 & & 0.306 & 0.0238 \\
10 & & 0.295 & 0.0245 \\
15 & & 0.292 & 0.0247 \\
\hline & (b) & & \\
\hline 2.5 & & 0.713 & 0.252 \\
5.5 & & 0.698 & 0.251 \\
10 & & 0.702 & 0.253 \\
\hline \hline
\end{tabular}


TABLE II. Comparison of $C_{L}$ and $C_{D}$ of the slender delta wing between experiments and calculation, with $\chi=76^{\circ}, R e=5 \times 10^{5}$, and $\alpha=20^{\circ}$.

\begin{tabular}{lccc}
\hline \hline Source & $\begin{array}{c}\text { Earnshaw and } \\
\text { Lawford }\end{array}$ & $\begin{array}{c}\text { Visser and } \\
\text { Washburm }\end{array}$ & $\begin{array}{c}\text { Present } \\
\text { calculation }\end{array}$ \\
\hline$C_{L}$ & 0.737 & 0.690 & 0.702 \\
$C_{D}$ & 0.239 & 0.243 & 0.253 \\
\hline \hline
\end{tabular}

that the difference of model geometries has only minor effect, see below.

In this study, a block structured grid was constructed by ICEMCFD. A boundary layer mesh was constructed around the elliptic wing and delta wing, with the height of the first layer being $y^{+} \approx 0.5$. Local mesh refinement was performed according to the peak position of the streamwise vorticity in the wake. An adaptive mesh technology based on vorticity magnitude was used. To test grid independence, the computed lift coefficient $C_{L}$ and drag coefficient $C_{D}$ with different grid numbers are compared in Table I. The results presented in the main text of this paper were calculated by the mesh with the finest grids.

The NASA experiment included a force measurement by balance with Wall-Pressure Signature Correction Method to eliminate the wind-tunnel-wall interface but without correction of support interference and a wake survey by 5 -hole probe hotwire anemometer. The survey area was found not big enough to yield lift and drag values comparable to those measured by balance. ${ }^{34}$ In our calculation, the range of wake plane location $x$ was in $x=(0,20)$ with origin at the wing apex. The comparison of $C_{L}$ and $C_{D}$ between the balance-measurement and calculation is shown in Table II.

The measured and computed distributions of velocity components over a wake plane $(\tilde{y}, \tilde{z})$ at $\tilde{x} / c=1.025$, where $(\tilde{x}, \tilde{y}, \tilde{z})$ are body axes, are compared in Fig. 17.

The location of the primary vortex and the shape of the vortex core match the experimental result very well, but the computed
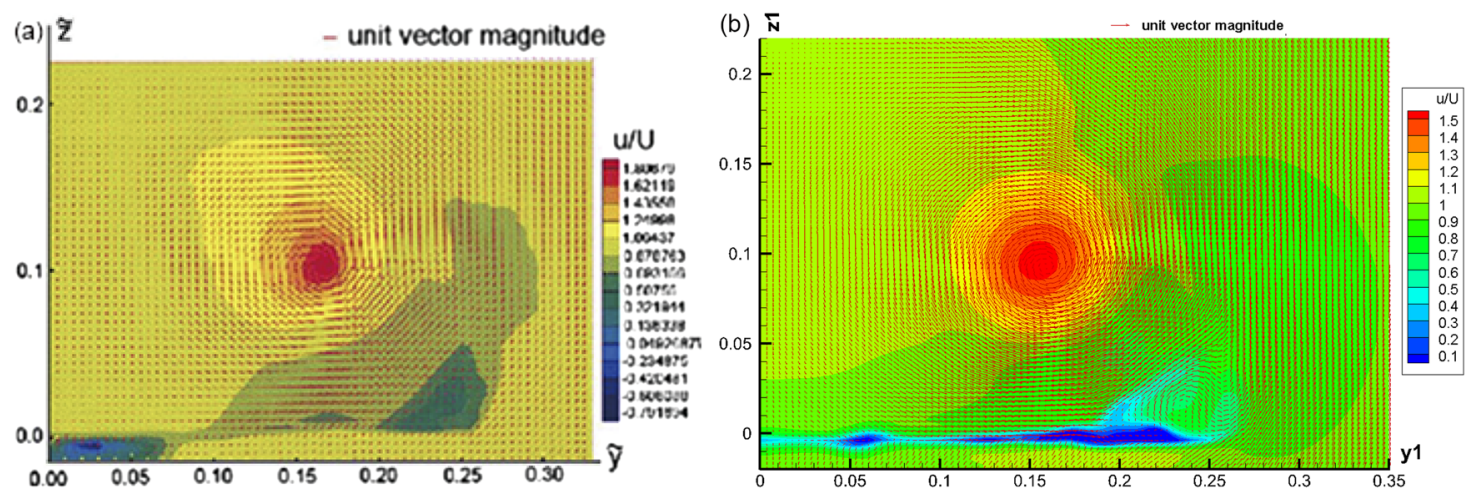

FIG. 17. Distributions of velocity components of the delta wing of a steady flow over a wake plane at $\tilde{x} / c=1.025$ (using body axes). $\chi=76^{\circ}, R e=5 \times 10^{5}$, and $\alpha=20^{\circ}$ (a) Measured data at NASA Langley by Visser and Washburm. The low axial velocity at the lower left corner was due to the interference of model support. (b) CFD data. Contours: $\tilde{u}$, vectors: $(\tilde{v}, \tilde{w})$, nondimensionalized by $U$.
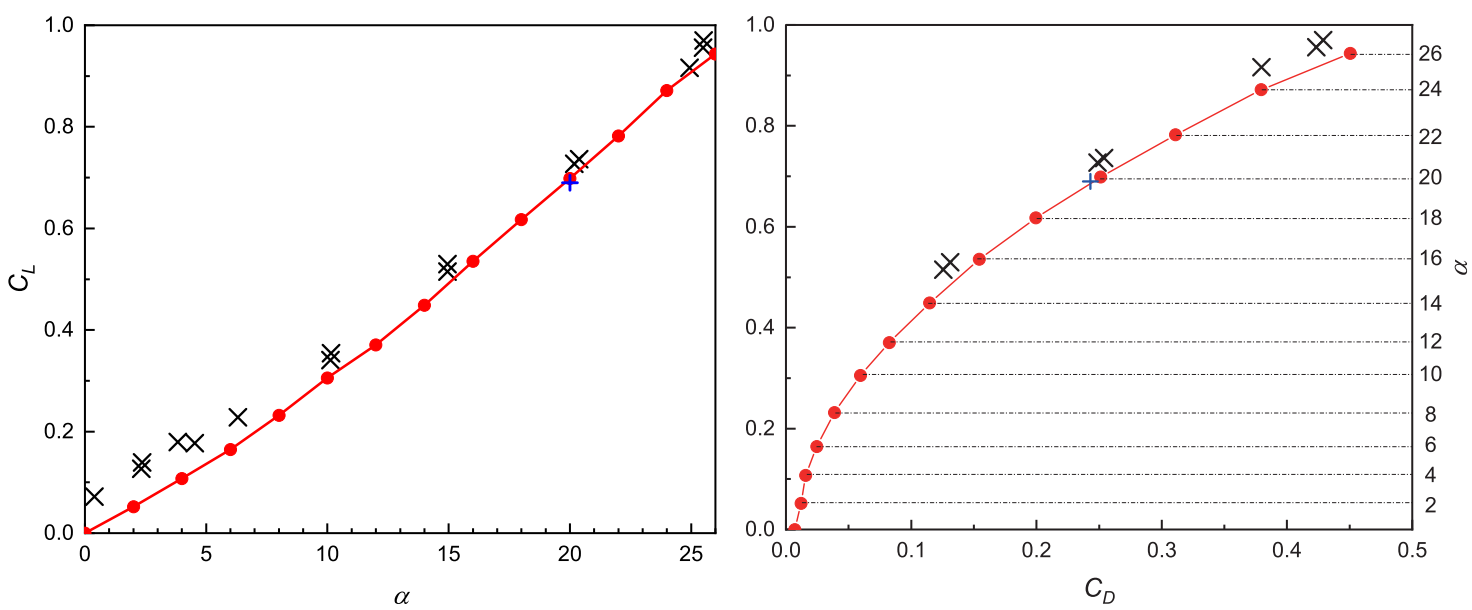

FIG. 18. Computed $C_{L}-\alpha$ curve (left) and lift-drag polar curve (right) compared with the experimental data for the same delta wing and Re as in Fig. 17. Present calculation: (red filled circle); EL experiment: (cross); NASA experiment: (plus). 

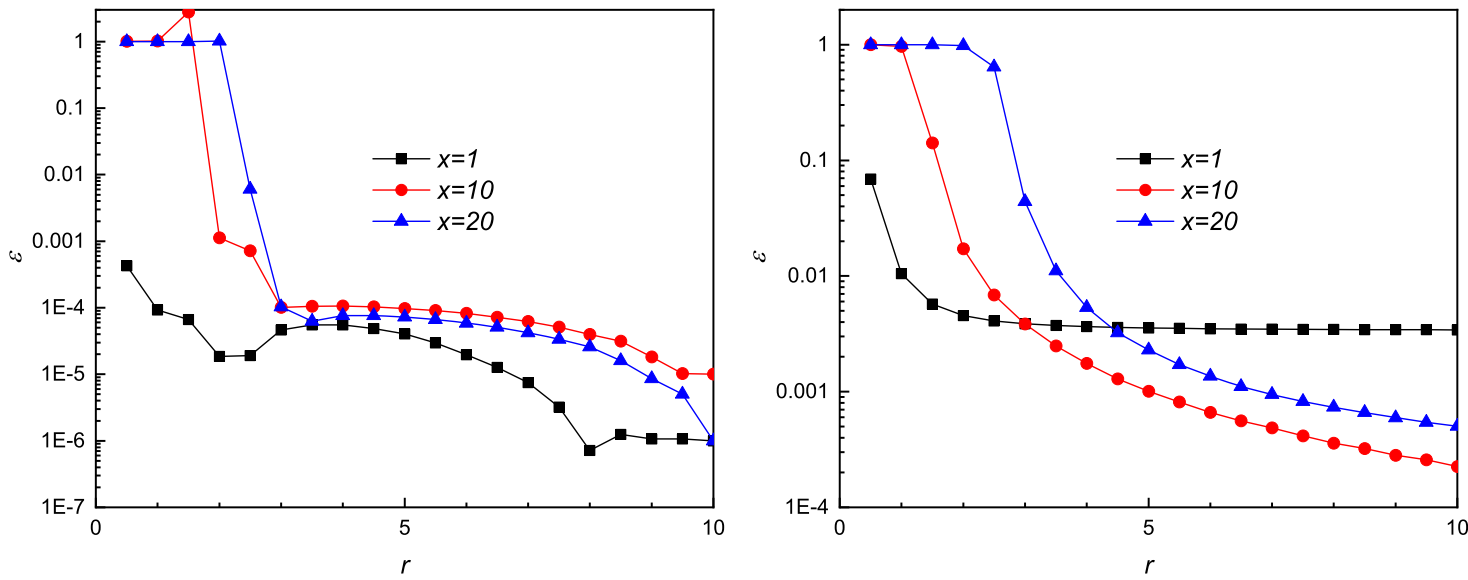

FIG. 19. The effect of size of integration domain for the delta wing. (Left) $\rho \int_{W_{v}} \boldsymbol{r} \cdot(\boldsymbol{v} \times \boldsymbol{\omega}) \mathrm{d} S$. (Right) $0.5 \rho \int_{W}\left(v^{2}+w^{2}-u^{\prime 2}\right) \mathrm{d} S$, with the same flow parameters as in Fig. 17 .

maximum $u$ at vortex centers is smaller than the experimental value likely due to the numerical dissipation.

The computed variation of lift and drag for different $\alpha^{\prime}$ s is compared with the EL experiment in Fig. 18. The computed $C_{L}$ curve deviates from the measured one at small $\alpha^{\prime}$, which seems to be mainly caused by the nonzero lift at $\alpha=0^{\circ}$ due to the asymmetry of the experimental model with a support.

The above validations indicate that the present calculation can predict the force and flow field structures very well.

\section{APPENDIX C: SOME DETAILED ISSUES OF CALCULATION}

\section{Effect of finite integration domain size}

In this paper, there are two kinds of $W$-integral based force components: compact and noncompact integrals. The sizes of the integration domain for these two $W$-integrals are different. The former only needs the vortical wake domain, and the latter needs the entire wake plane. In calculation, the wake vortex moves downward and spreads along streamwise due to the downwash and viscosity, respectively. If the vortical wake is identified for each wake plane, the size for compact vortical $W_{v}$-integrals is minimal, but it requires extra calculations. At the same time, to obtain all information on the entire wake plane is impossible. Therefore, our strategy is to check the $W$-integral as a function of integration domain size and then find the suitable integration domain within an acceptable error. The criterion is defined by the relative error

$$
\epsilon=\left|\frac{f(r)-f_{\infty}}{f_{\infty}}\right|,
$$

where $f(r)$ is the $W$-integral value as a function of the size, $r$ is the radius of the circular integration domain, and $f_{\infty}$ is the reference value obtained by integrating over the entire calculation plane.

Integrals $\rho \int_{W_{v}} \boldsymbol{r} \cdot(\boldsymbol{v} \times \boldsymbol{\omega}) \mathrm{d} S$ and $0.5 \rho \int_{W}\left(v^{2}+w^{2}-u^{\prime 2}\right) \mathrm{d} S$ are selected as the representatives of the compact and noncompact integrals, respectively. The relative errors on three different wake planes in a typical model flow are shown in Fig. 19. In order to demonstrate the difference clearly, the ordinate uses logarithmic coordinates.

For compact $W_{v}$-integrals as shown in Fig. 19 (left), the relative error is less than $10^{-4}$ when $r>3$ on any wake plane. Therefore, a circular integration domain of $r=3 c$ is selected for the calculation of compact $W_{v}$-integrals. For noncompact $W$-integrals as shown in Fig. 19 (right), the relative error is one to three orders of magnitude larger than that of $W_{v}$-integrals. Moreover, the relative error is difficult to reduce near the trailing edge due to the dispersion of the disturbance terms. The circle with $r=10 c$ is chosen as the integration domain to reduce the calculated amount and ensure the reliability of most wake planes.

\section{Viscous term on the $W$}

In calculation, the effect of shear stress $\tau$ in the wake plane is very small and not plotted in figures. For the sake of rigor, the

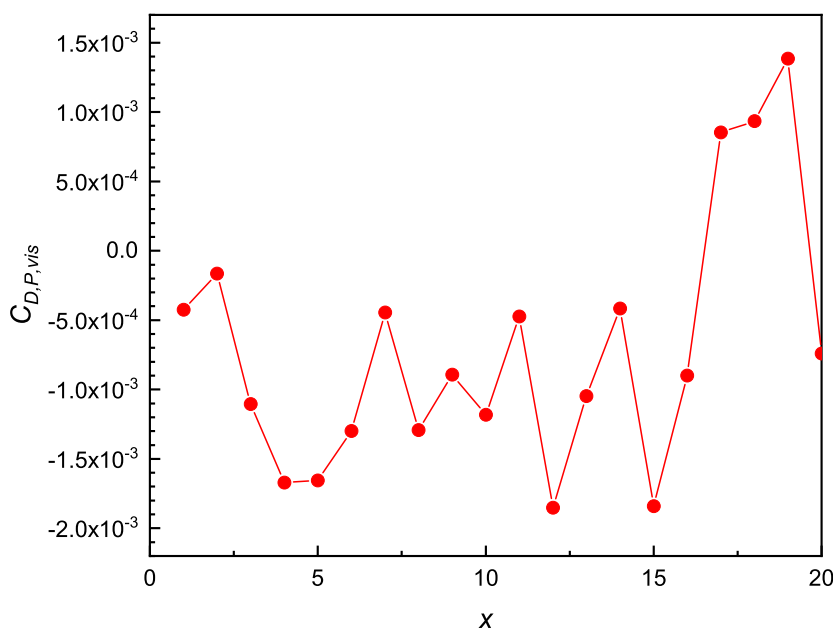

FIG. 20. The $x$-dependency of viscous terms in $D_{P}$ for the delta wing, with the same flow parameters as in Fig. 17. 
value of the viscous term of (11) will be presented in detail here. The Reynolds numbers of the two wings are of the same magnitude, but the separated flow is more complicated, and the viscous effect near the trailing edge is also greater. The results of the delta wing at $\alpha=20^{\circ}$ are shown in Fig. 20. The viscous term is a few orders of magnitude smaller than $D_{P}$ and can therefore be completely ignored.

\section{REFERENCES}

${ }^{1}$ L. Prandtl, “Tragflügeltheorie. I. Mitteilung," Nachr. Ges. Wiss. Goettingen, Math.-Phys. Kl. 1918, 451-477.

${ }^{2}$ A. Betz, "A method for the direct determination of wing-section drag," Report No. NACA-TM-337, NACA, 1925.

${ }^{3}$ G. I. Taylor, "Note on the connection between the lift on an aërofoil in a wind and the circulation round it," Philos. Trans. R. Soc., A 225, 238-246 (1926).

${ }^{4}$ B. W. McCormick, Aerodynamics, Aeronautics, and Flight Mechanics (Wiley, New York, 1995), Vol. 2.

${ }^{5}$ J. P. Marec, "Drag reduction: A major task for research," in Aerodynamic Drag Reduction Technologies (Springer, 2001), pp. 17-27.

${ }^{6} \mathrm{~K}$. Kusunose, A Wake Integration Method for Airplane Drag Prediction (Tohoku University Press, Sendai, 2005).

${ }^{7}$ G. K. Batchelor, An Introduction to Fluid Dynamics (Cambridge University Press, Cambridge, 1967).

${ }^{8}$ J. Lighthill, "Fluid mechanics," in Twentieth Century Physics (AIP Press, New York, 1995), pp. 795-912, Book Section 10.

${ }^{9}$ P. R. Spalart, "On the far wake and induced drag of aircraft," J. Fluid Mech. 603, 413-430 (2008).

${ }^{10}$ J. E. Yates and C. D. Donaldson, "A fundamental study of drag and an assessment of conventional drag-due-to-lift reduction devices," Report No. NASA-CR4004, 1986.

${ }^{11}$ R. Cummings, M. Giles, and G. Shrinivas, "Analysis of the elements of drag in three-dimensional viscous and inviscid flows," AIAA Paper No. 96-2482-CP, 1996.

${ }^{12}$ D. D. Chao and C. P. van Dam, "Wing drag prediction and decomposition," J. Aircr. 43, 82-90 (2006).

${ }^{13}$ M. Méheut and D. Bailly, "Drag-breakdown methods from wake measurement," AIAA J. 46, 847-862 (2008).

${ }^{14}$ L. Q. Liu, J. Z. Wu, W. D. Su, and L. L. Kang, "Lift and drag in three-dimensional steady viscous and compressible flow," Phys. Fluids 29, 116105 (2017).

${ }^{15}$ C. P. van Dam, "Recent experience with different methods of drag prediction," Prog. Aerosp. Sci. 35, 751-798 (1999).

${ }^{16}$ D. Hunt, R. Cummings, and M. Giles, "Determination of drag from threedimensional viscous and inviscid flow field computations," AIAA Paper No. 97-2257, 1997.

${ }^{17}$ V. Schmitt and D. Destarac, "Recent progress in drag prediction and reduction for civil transport aircraft at onera," AIAA Paper No. 98-137, 1998.

${ }^{18}$ T. Snyder and A. Povitsky, "Far-field induced drag prediction using vorticity confinement technique," J. Aircr. 51, 1953-1958 (2014).

${ }^{19}$ Y. T. Fan and W. P. Li, "Review of far-field drag decomposition methods for aircraft design,” J. Aircr. 56, 11-21 (2018).
${ }^{20}$ J. van der Vooren and J. W. Slooff, "CFD-based drag prediction; State-of-theart, theory, prospects," in Lecture Notes of AIAA Professional Studies Series, NLR TP 90247, 1990.

${ }^{21}$ C. Marongiu and R. Tognaccini, "Far-field analysis of the aerodynamic force by Lamb vector integrals," AIAA J. 48, 2543-2555 (2010).

${ }^{22}$ C. Marongiu, R. Tognaccini, and M. Ueno, "Lift and lift-induced drag computation by Lamb vector integration," AIAA J. 51, 1420-1430 (2013).

${ }^{23}$ B. Mele and R. Tognaccini, "Aerodynamic force by Lamb vector integrals in compressible flow,” Phys. Fluids 26, 056104 (2014).

${ }^{24} \mathrm{H}$. Glauert, The Elements of Aerofoil and Airscrew Theory, 2nd ed. (Cambridge University Press, Cambridge, 1947).

${ }^{25}$ E. C. Maskell, "Progress towards a method for the measurement of the components of the drag of a wing of finite span," Report No. RAE TP 72232, Royal Aircraft Establishment, 1972.

${ }^{26}$ M. B. Giles and R. M. Cummings, "Wake integration for three-dimensional flow field computations: Theoretical development," J. Aircr. 36, 357-365 (1999).

${ }^{27}$ D. Destarac and J. van der Vooren, "Drag/thrust analysis of jet-propelled transonic transport aircraft; Definition of physical drag components," Aerosp. Sci. Technol. 8, 545-556 (2004).

${ }^{28}$ J. Z. Wu, L. Q. Liu, and T. S. Liu, "Fundamental theories of aerodynamic force in viscous and compressible complex flows," Prog. Aerosp. Sci. 99, 27-63 (2018).

${ }^{29}$ J. Z. Wu, H. Y. Ma, and M. D. Zhou, Vorticity and Vortex Dynamics (Springer Science \& Business Media, 2006).

${ }^{30}$ J. Z. Wu, X. Y. Lu, and L. X. Zhuang, "Integral force acting on a body due to local flow structures,” J. Fluid Mech. 576, 265-286 (2007).

${ }^{31}$ Y. T. Yang, R. K. Zhang, Y. R. An, and J. Z. Wu, "Steady vortex force theory and slender-wing flow diagnosis," Acta Mech. Sin. 23, 609-619 (2007).

${ }^{32}$ C. P. van Dam, K. Nikfetrat, P. M. H. W. Vijgen, and C. M. Fremaux, "Calculation and measurement of induced drag at low speeds," Report No. SAE Technical Paper 901935, SAE, 1990.

${ }^{33}$ P. B. Earnshaw and J. A. Lawford, Low-Speed Wind-Tunnel Experiments on a Series of Sharp-Edged Delta Wings (HM Stationery Office London, 1966).

${ }^{34}$ J. M. Wu, B. Ondrusek, and J. Z. Wu, "Exact force diagnostics of vehicles based on wake-plane data," AIAA Paper No. 96-0559, 1996.

${ }^{35} \mathrm{H}$. Toubin and D. Bailly, "Development and application of a new unsteady farfield drag decomposition method," AIAA J. 53, 3414-3429 (2015).

${ }^{36}$ L. L. Kang, L. Russo, R. Tognaccini, J. Z. Wu, and W. D. Su, "Aerodynamic force breakdown based on vortex force theory,” AIAA Paper 2019-2122, 2019.

${ }^{37}$ L. W. Bryant and D. H. Williams, "An investigation of the flow of air around an aerofoil of infinite span,” Philos. Trans. R. Soc., A 225, 199-245 (1926).

${ }^{38}$ J. Z. Wu, H. Y. Ma, and M. D. Zhou, Vortical Flows (Springer, 2015).

${ }^{39}$ L. Q. Liu, Unified Theoretical Foundations of Lift and Drag in Viscous and Compressible External Flows (Springer, Singapore, 2018).

${ }^{40}$ D. W. Moore and P. G. Saffman, "Axial flow in laminar trailing vortices," Proc. R. Soc. London, Ser. A 333, 491-508 (1973).

${ }^{41}$ J. Serrin, "Mathematical principles of classical fluid mechanics," in Fluid Dynamics I/Strömungsmechanik I (Springer, 1959), pp. 125-263.

${ }^{42}$ M. Ueno, K. Yamamoto, K. Tanaka, M. Murayama, and R. Tognaccini, "Farfield drag analysis of nasa common research model simulation," J. Aircr. 50, 388-397 (2013).

${ }^{43}$ H. Lamb, Hydrodynamics (Cambridge University Press, 1932).

${ }^{44} \mathrm{H}$. Helmholtz, "On integrals of the hydrodynamical equations which express vortex-motion,” J. Reine Angew. Math. 55, 25-55 (1858). 